\title{
Structural Revision of the Hancock Alkaloid (-)-Galipeine
}

\author{
Stephen G. Davies,* Ai M. Fletcher, Ian T. T. Houlsby, Paul M. Roberts, and James E. Thomson \\ Department of Chemistry, Chemistry Research Laboratory, University of Oxford, Mansfield Road, Oxford OX1 3TA, U.K. \\ Supporting Information Placeholder
}
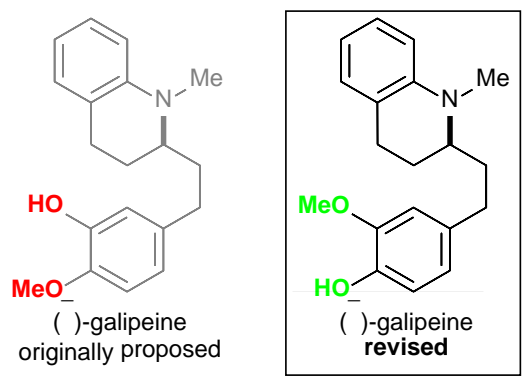

\begin{abstract}
The ${ }^{1} \mathrm{H}$ and ${ }^{13} \mathrm{C}$ NMR data of synthetic samples of $(S)-N(1)$-methyl-2-[2'-(3"-hydroxy-4"-methoxyphenyl)ethyl]1,2,3,4-tetrahydroquinoline, the originally proposed structure of the Hancock alkaloid (-)-galipeine, do not match those of the natural product. Herein, the preparation of the regioisomer $(S)-N(1)$-methyl-2-[2'-(3"-methoxy-4"-hydroxyphenyl)ethyl]-1,2,3,4tetrahydroquinoline is reported, the ${ }^{1} \mathrm{H}$ and ${ }^{13} \mathrm{C}$ NMR data of which are in excellent agreement with those of $(-)$-galipeine. Comparison of specific rotation data enables assignment of the absolute $(S)$-configuration of the alkaloid and together, these data engender the structural revision of (-)-galipeine to $(S)-N(1)$-methyl-2-[2'-(3"-methoxy-4"-hydroxyphenyl)ethyl]-1,2,3,4-tetrahydroquinoline.
\end{abstract}

The isolation of the alkaloid (-)-galipeine from the angostura (trunk bark) of Galipea officinalis Hancock was first reported by Jacquemond-Collet et al. in 1999., ${ }^{1,2}$ Following interrogation by NMR spectroscopy and mass spectrometry, it was assigned the gross structure of $N(1)$-methyl-2-[2'-(3"-hydroxy4"-methoxyphenyl)ethyl]-1,2,3,4-tetrahydroquinoline (its absolute configuration was not determined). ${ }^{1}(-)$-Galipeine together with (-)-angustureine, (-)-cuspareine and (-)galipinine (Figure 1) comprise a small family of congeneric angostura alkaloids based on a 2-substituted 1,2,3,4tetrahydroquinoline core, which are popular targets for laboratory syntheses. ${ }^{3-10}$ However, galipeine has received scant attention from the synthetic community compared to the remainder of the tetrad: more than ten syntheses of each of the other alkaloids have been reported, whilst only two studies concerning galipeine have appeared to date. Zhou et al. were first to report their synthesis of enantiopure $(S)-N(1)$-methyl-2[2'-(3"-hydroxy-4"-methoxyphenyl)ethyl]-1,2,3,4-

tetrahydroquinoline [possessing the gross structure assigned to (-)-galipeine] in 2004, ${ }^{11}$ and concluded that the ${ }^{1} \mathrm{H}$ and ${ }^{13} \mathrm{C}$ NMR data of this material were identical to those of the natural product. Comparison of their specific rotation value of $[\alpha]_{\mathrm{D}}{ }^{20}-26.1$ (c 0.44 in $\left.\mathrm{CHCl}_{3}\right)^{12}$ with that reported for the natural product of $[\alpha]_{\mathrm{D}}-13.6$ (temperature, concentration nor solvent were specified) ${ }^{1}$ led Zhou et al. to propose the absolute (S)-configuration for the natural product. ${ }^{11}$ Subsequently, Hii et al. reported an alternative approach to $(S)-N(1)$-methyl-2[2'-(3"-hydroxy-4"-methoxyphenyl)ethyl]-1,2,3,4-

tetrahydroquinoline in $2012^{13}$ and asserted the congruency of their synthetic sample with the natural product by comparison of NMR data and sign of the specific rotation value. In fact, inspection of the ${ }^{1} \mathrm{H}$ and ${ }^{13} \mathrm{C}$ NMR data reported for (-)galipeine with those of both Zhou et al. ${ }^{11}$ and Hii et al. ${ }^{13}$ for their synthetic samples of $(S)-N(1)$-methyl-2-[2'-(3"'-hydroxy4"-methoxyphenyl)ethyl]-1,2,3,4-tetrahydroquinoline shows some discrepancies. In this manuscript, we report the preparation of an independent sample of $(S)-N(1)$-methyl-2-[2'-(3"hydroxy-4"-methoxyphenyl)ethyl]-1,2,3,4-tetrahydroquinoline by a new route involving conjugate addition of lithium $(R)-N$ benzyl- $N$-( $\alpha$-methyl- $p$-methoxybenzyl)amide to 4-(obromophenyl)- $N$-methoxy- $N$-methylpent-2-enamide to set the stereochemistry, and use of a Buchwald-Hartwig coupling reaction to construct the 1,2,3,4-tetrahydroquinoline core. The ${ }^{1} \mathrm{H}$ and ${ }^{13} \mathrm{C}$ NMR data (as well as the specific rotation value) of our sample of this material are in accord with those of Zhou et al. ${ }^{11}$ and Hii et al. ${ }^{13}$ but these data do not match those for the natural product (-)-galipeine, as expected. ${ }^{1}$ A more detailed analysis of the available data led us to propose that (-)galipeine is in fact the regioisomeric compound $N(1)$-methyl2-[2'-(3"-methoxy-4"-hydroxyphenyl)ethyl]-1,2,3,4-

tetrahydroquinoline. We prepared an authentic, enantiopure sample of this material and its ${ }^{1} \mathrm{H}$ and ${ }^{13} \mathrm{C}$ NMR data were found to be effectively identical to those of the natural product. Comparison of specific rotation data allowed us to assign the absolute $(S)$-configuration to (-)-galipeine. Together, these data thus call for the structural revision of (-)-galipeine to $(S)$ $N(1)-m e t h y l-2-[2 '-(3 "$-methoxy-4"-hydroxyphenyl)ethyl]-

1,2,3,4-tetrahydroquinoline and the studies contained herein comprise the unambiguous structural determination of (-)galipeine, its first asymmetric synthesis, and assignment of its absolute configuration. 
<smiles>CCCCCC1CCc2ccccc2N1C</smiles>

( )-angustureine

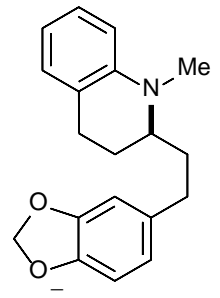

( )-galipinine

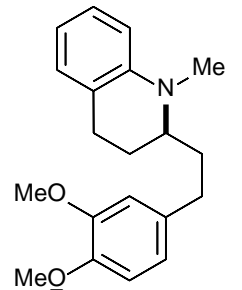

( )-cuspareine

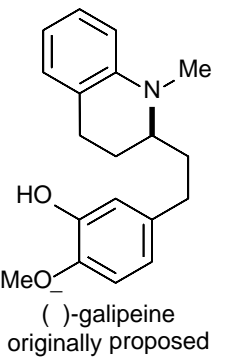

Figure 1. Structures of tetrahydroquinoline Hancock alkaloids.

Alerted to discrepancies in the reported data, our initial goal was the preparation of an independent sample of $(S)-N(1)$ methyl-2-[2'-(3"-hydroxy-4"-methoxyphenyl)ethyl]-1,2,3,4tetrahydroquinoline [the structure originally assigned to (-)galipeine]. ${ }^{1}$ Our synthesis commenced from commercially available 3-(o-bromophenyl)propanoic acid 1. Reduction of $\mathbf{1}$ using $\mathrm{NaBH}_{4}$ in the presence of $\mathrm{BF}_{3} \cdot \mathrm{OEt}_{2}{ }^{14}$ gave the corresponding alcohol 2 in 92\% yield. One-pot Swern oxidation/Wittig reaction of 2 using $\mathrm{Ph}_{3} \mathrm{P}=\mathrm{CHCON}(\mathrm{Me})(\mathrm{OMe}) \mathbf{3}$ as the ylide then gave 4 as a single diastereoisomer [ $>95: 5 \mathrm{dr}$, $(E):(Z)$ ratio], which was isolated in $78 \%$ yield. The geometry of the olefin within 4 was assigned on the basis of the diagnostic ${ }^{1} \mathrm{H}$ NMR ${ }^{3} J$ coupling constant: ${ }^{3} J_{\mathrm{H}-2, \mathrm{H}-3}=15.4 \mathrm{~Hz}$. Conjugate addition of lithium $(R)-N$-benzyl- $N$-( $\alpha$-methyl- $p$ methoxybenzyl)amide 5 then delivered $\beta$-amino amide $\mathbf{6}$ as a single diastereoisomer ( $>95: 5 \mathrm{dr}$ ), which was isolated in $80 \%$ yield. The absolute configuration of the newly formed C(3)stereogenic center of $\mathbf{6}$ was assigned by reference to the transition state mnemonic devised by us to predict the stereochemical outcome of this type of conjugate addition reaction. ${ }^{15}$ Subsequent treatment of $\mathbf{6}$ with $\mathrm{HCO}_{2} \mathrm{H}$ in the presence of $\mathrm{Et}_{3} \mathrm{SiH}^{16-18}$ effected chemoselective removal of the $\mathrm{N}$ - $\alpha$ methyl-p-methoxybenzyl group to furnish 7 in $81 \%$ yield. Treatment of 7 with $5 \mathrm{~mol} \% \mathrm{Pd}(\mathrm{OAc})_{2}$ in the presence of XPhos and $\mathrm{Cs}_{2} \mathrm{CO}_{3}$ in PhMe at reflux for $24 \mathrm{~h}$ gave tetrahydroquinoline $\mathbf{8}$ quantitatively. O-tert-Butyldimethylsilyl-5bromoguaiacol 9 was converted to the corresponding aryllithium regent $\mathbf{1 0}$ upon reaction with $n-\mathrm{BuLi}$; addition of $\mathbf{8}$ to the reaction flask then produced ketone $\mathbf{1 1}$ after work-up, which was isolated in $88 \%$ yield. Treatment of $\mathbf{1 1}$ with $\mathrm{LiAlH}_{4}$ and then TFA in the presence of $\mathrm{Et}_{3} \mathrm{SiH}$ gave 12 in $50 \%$ yield (i.e., in addition to reduction of the ketone functionality, deprotection of the $O$-silyl group had also occurred during this twostep sequence). Subjection of $\mathbf{1 2}$ to an atmosphere of hydrogen in the presence of $\mathrm{Pd} / \mathrm{C}$ and aqueous formaldehyde (formalin) resulted in hydrogenolytic $N$-debenzylation and reductive $N$ methylation, giving 13 in 57\% yield (Scheme 1). The identity of 13 was unambiguously secured by single crystal X-ray diffraction analysis, ${ }^{19}$ and the determination of a Flack $x$ parame$\operatorname{ter}^{20,21}$ for the crystal structure of $-0.1(2)$, which satisfies the criterion for reliable configurational assignment of a material known to be enantiopure, confirmed its absolute $(S)$ - configuration. Thus, the absolute configurations that had been assigned to the precursors 6-8, 11 and $\mathbf{1 2}$ were also confirmed (Figure 2).

Scheme 1. (S)-N(1)-Methyl-2-[2'-(3"-hydroxy-4"methoxyphenyl)ethyl]-1,2,3,4-tetrahydroquinoline 13

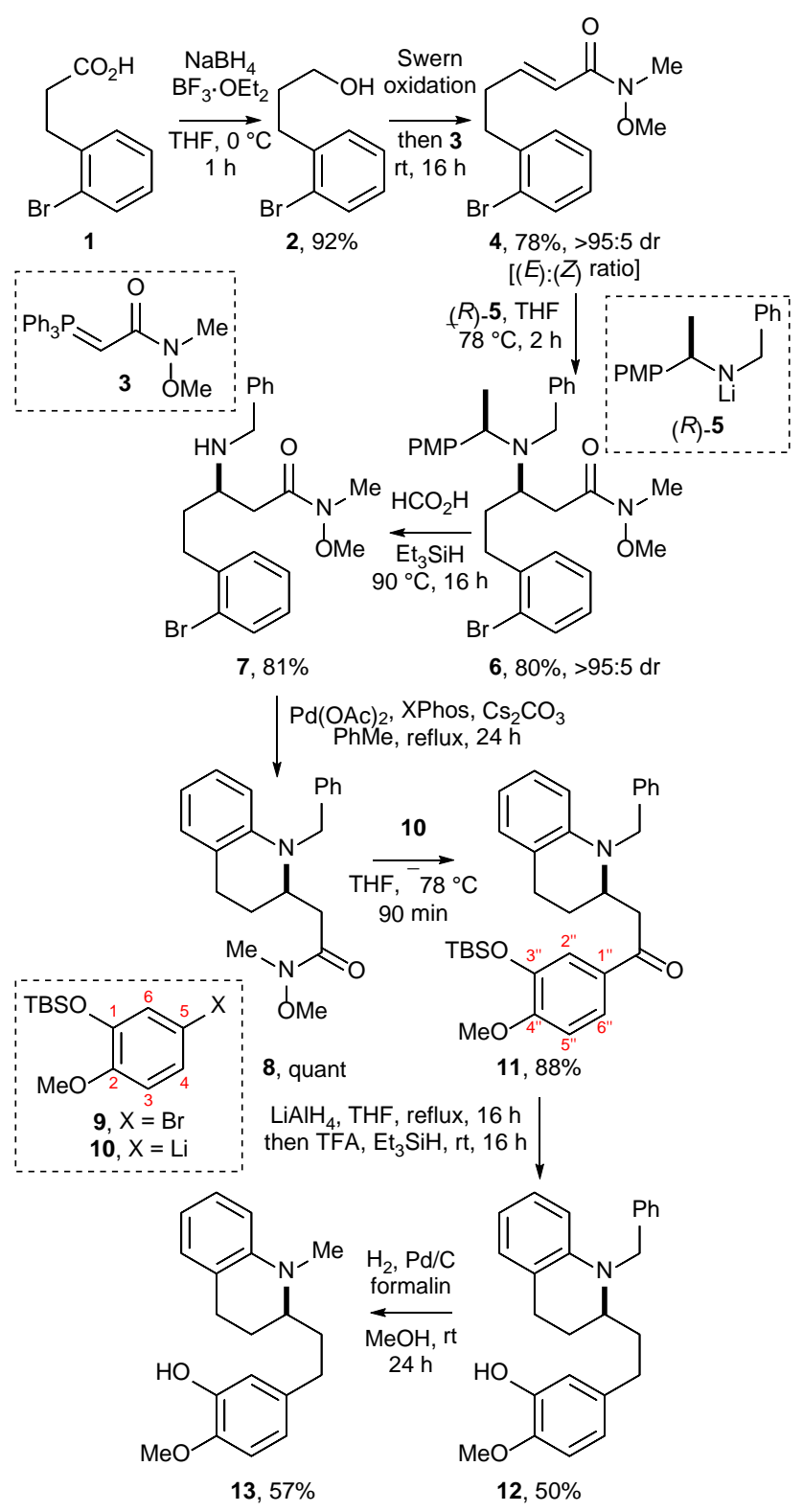

PMP = p-methoxyphenyl. TBS = tert-butyldimethylsilyl.

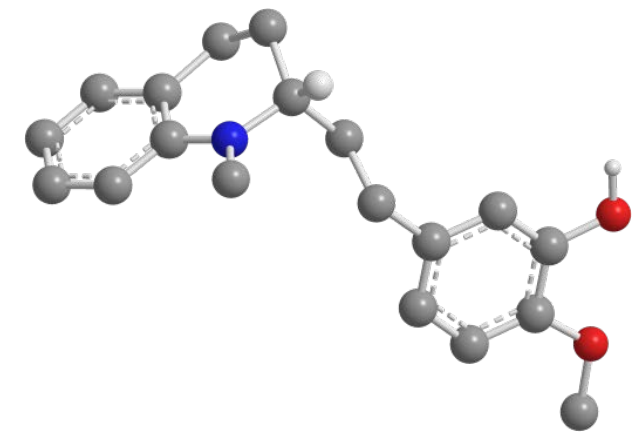

Figure 2. Chem3D representation of the X-ray crystal structure of (S)-13 (selected $\mathrm{H}$ atoms are omitted for clarity). 
The specific rotation of our sample of $\mathbf{1 3}$ was $[\alpha]_{\mathrm{D}}{ }^{25}-26.2$ (c 1.0 in $\mathrm{CHCl}_{3}$ ) whilst Zhou et al. measured $[\alpha]_{\mathrm{D}}{ }^{20}-26.1$ (c 0.44 in $\left.\mathrm{CHCl}_{3}\right)^{11,12}$ and Hii et al. obtained $[\alpha]_{\mathrm{D}}^{25}-27.0$ (c 0.7 in $\left.\mathrm{CHCl}_{3}\right),{ }^{13}$ demonstrating truly remarkable parity. The ${ }^{13} \mathrm{C}$ NMR data of all three samples also display excellent agreement $\left(\Delta \delta_{\mathrm{C}} \leq 0.2 \mathrm{ppm}\right),{ }^{22}$ as do the ${ }^{1} \mathrm{H}$ NMR data of our sample of 13 and those of Hii et al. $\left(\Delta \delta_{\mathrm{H}} \leq 0.05 \mathrm{ppm}\right) .{ }^{22}$ Our ${ }^{1} \mathrm{H}$ NMR chemical shift values of $\mathbf{1 3}$ are in very good accord with those reported by Zhou et al. $\left(\Delta \delta_{\mathrm{H}} \leq 0.04 \mathrm{ppm}\right)^{22}$ although the relative integrations of some of the multiplets below $\sim 2.7 \mathrm{ppm}$ are not the same. There are, however, clearly issues associated with the relative integrations of the ${ }^{1} \mathrm{H}$ NMR data presented by Zhou et al. as twenty-four protons are listed in total ${ }^{11}$ (the formula of 13 being $\mathrm{C}_{19} \mathrm{H}_{23} \mathrm{NO}_{2}$ ). Notwithstanding these differences, it is unequivocal that the three samples of $(S)-N(1)$ methyl-2-[2'-(3"-hydroxy-4"-methoxyphenyl)ethyl]-1,2,3,4tetrahydroquinoline $\mathbf{1 3}$ are the same. ${ }^{23}$

Concerning comparison of the ${ }^{1} \mathrm{H}$ and ${ }^{13} \mathrm{C}$ NMR data of $\mathbf{1 3}$ with those of (-)-galipeine, we must first express our gratitude to Professor Nicolas Fabre who supplied us with copies of the NMR spectra of (-)-galipeine for this purpose. Unfortunately, some transcription errors (mainly concerning determination of the relative integrations of the multiplets below $\sim 2.7 \mathrm{ppm}$ ) were noted when the raw ${ }^{1} \mathrm{H}$ NMR spectrum of (-)-galipeine was compared to the reported data. ${ }^{1}$ We therefore first corrected the ${ }^{1} \mathrm{H}$ NMR data of (-)-galipeine from the original spectrum (Figure 3). Whilst casual inspection of the ${ }^{1} \mathrm{H}$ NMR data of 13 and (-)-galipeine indicates broad agreement, more careful analysis reveals that $\mathbf{1 3}$ shows a $1 \mathrm{H}$ multiplet at $6.66 \mathrm{ppm}$ and a $2 \mathrm{H}$ multiplet at $6.77 \mathrm{ppm}$; in (-)-galipeine the relative integrations associated with the analogous signals are transposed. Perhaps less subtle, the ${ }^{13} \mathrm{C}$ NMR data displays $\Delta \delta_{\mathrm{C}} \geq$ $1.0 \mathrm{ppm}^{22}$ for four of the carbon atoms. Such large differences clearly suggest that the structures of $\mathbf{1 3}$ and (-)-galipeine are not the same, and it followed that the structure of the alkaloid had been erroneously assigned (Figure 3 ).<smiles>COc1ccc(CC[C@@H]2CCc3ccccc3N2C)cc1O</smiles>

${ }^{1} \mathrm{H}$ NMR (400 MHz, $\mathrm{CDCl}_{3}$ ) ${ }^{13} \mathrm{C}$ NMR (100 MHz, $\left.\mathrm{CDCl}_{3}\right)$

\begin{tabular}{l|l}
\hline 13 & ( )-galipeine \\
\hline $1.71(1 \mathrm{H}, \mathrm{m})$ & $1.70(1 \mathrm{H}, \mathrm{m})^{\mathrm{a}}$ \\
$1.92(3 \mathrm{H}, \mathrm{m})$ & $1.90(3 \mathrm{H}, \mathrm{m})^{\mathrm{a}}$ \\
$2.49(1 \mathrm{H}, \mathrm{ddd})$ & $2.50(1 \mathrm{H}, \mathrm{m})^{\mathrm{a}}$ \\
$2.66(2 \mathrm{H}, \mathrm{m})$ & $2.68(2 \mathrm{H}, \mathrm{m})^{\mathrm{a}}$ \\
$2.84(1 \mathrm{H}, \mathrm{ddd})$ & $2.82(1 \mathrm{H}, \mathrm{m})$ \\
$2.90(3 \mathrm{H}, \mathrm{s})$ & $2.89(3 \mathrm{H}, \mathrm{s})$ \\
$3.27(1 \mathrm{H}, \mathrm{m})$ & $3.26(1 \mathrm{H}, \mathrm{m})$ \\
$3.87(3 \mathrm{H}, \mathrm{s})$ & $3.85(3 \mathrm{H}, \mathrm{s})$ \\
$5.56(1 \mathrm{H}, \mathrm{s})$ & $5.45(1 \mathrm{H}, \mathrm{s})^{\mathrm{a}}$ \\
$6.52(1 \mathrm{H}, \mathrm{d})$ & $6.51(1 \mathrm{H}, \mathrm{d})$ \\
$6.59(1 \mathrm{H}, \mathrm{td})$ & $6.57(1 \mathrm{H}, \mathrm{td})$ \\
$6.66(1 \mathrm{H}, \mathrm{dd})$ & $6.65(2 \mathrm{H}, \mathrm{m})$ \\
$6.77(2 \mathrm{H}, \mathrm{m})$ & $6.80(1 \mathrm{H}, \mathrm{d})$ \\
$6.98(1 \mathrm{H}, \mathrm{d})$ & $6.96(1 \mathrm{H}, \mathrm{d})$ \\
$7.08(1 \mathrm{H}, \mathrm{td})$ & $7.06(1 \mathrm{H}, \mathrm{t})$ \\
\hline
\end{tabular}

\begin{tabular}{c|c}
\hline $\mathbf{1 3}$ & ( )-galipeine \\
\hline 23.5 & $23.6(0.1)$ \\
24.3 & $24.4(0.1)$ \\
31.6 & $32.1(0.5)$ \\
32.9 & $33.2(0.3)$ \\
38.0 & $38.2(0.2)$ \\
\hline 56.0 & $56.0(0.0)$ \\
58.2 & $58.5(0.3)$ \\
\hline 110.5 & $110.7(0.2)$ \\
110.6 & $110.8(0.2)$ \\
114.5 & $114.3(0.2)$ \\
\hline 115.3 & $115.4(0.1)$ \\
119.5 & $120.9(1.4)$ \\
121.7 & $121.8(0.1)$ \\
\hline 127.1 & $127.2(0.1)$ \\
128.6 & $128.8(0.2)$ \\
\hline 135.3 & $134.0(1.3)$ \\
\hline 144.7 & $143.7(1.0)$ \\
\hline 145.3 & $145.4(0.1)$ \\
145.5 & $146.5(1.0)$ \\
\hline
\end{tabular}

Figure 3. ${ }^{1} \mathrm{H}$ and ${ }^{13} \mathrm{C}$ NMR data of 13 and (-)-galipeine. Midpoints of all multiplets are quoted. Values of $\Delta \delta_{\mathrm{C}}$ are given in parentheses. ${ }^{\text {aThese }}{ }^{1} \mathrm{H}$ NMR data of $(-)$-galipeine are corrected here (compared to those reported in Ref 1 ) by analysis of the ${ }^{1} \mathrm{H}$ NMR spectrum of the natural product.

Further analysis of the ${ }^{1} \mathrm{H}$ and ${ }^{13} \mathrm{C}$ NMR data of $\mathbf{1 3}$ and the data reported for $(-)$-galipeine ${ }^{1}$ revealed a likely origin of the structural misassignment. Much of the ${ }^{1} \mathrm{H}$ and ${ }^{13} \mathrm{C}$ NMR data of the two compounds is effectively identical and is entirely consistent with them both sharing a 2-substituted N(1)-methyl1,2,3,4-tetrahydroquinoline moiety, whilst the variances suggest that differentially substituted aryl rings cap the C(2)-side chain. As the locations of the hydroxy and methoxy substituents within (-)-galipeine were assigned on the basis of the interpretation of its ${ }^{1} \mathrm{H}-{ }^{13} \mathrm{C}$ HMBC NMR spectrum, ${ }^{1}$ we proposed that (-)-galipeine is in fact the regioisomer $(S)-N(1)$ methyl-2-[2'-(3"-methoxy-4"-hydroxyphenyl)ethyl]-1,2,3,4tetrahydroquinoline 18. In order to investigate this hypothesis, an authentic sample of $\mathbf{1 8}$ was prepared. O-tertButyldimethylsilyl-4-bromoguaiacol 14 was converted to the aryllithium regent 15, which reacted with $\mathbf{8}$ to give ketone $\mathbf{1 6}$ in $62 \%$ yield. Sequential treatment of $\mathbf{1 6}$ with $\mathrm{LiAlH}_{4}$ and then TFA and $\mathrm{Et}_{3} \mathrm{SiH}$ gave 17 in 62\% yield. Finally, hydrogenolysis of $\mathbf{1 7}$ in the presence of formalin gave $\mathbf{1 8}$ in $70 \%$ yield (Scheme 2).

\section{Scheme 2. (S)-N(1)-Methyl-2-[2'-(3"-methoxy-4"-} hydroxyphenyl)ethyl]-1,2,3,4-tetrahydroquinoline 18

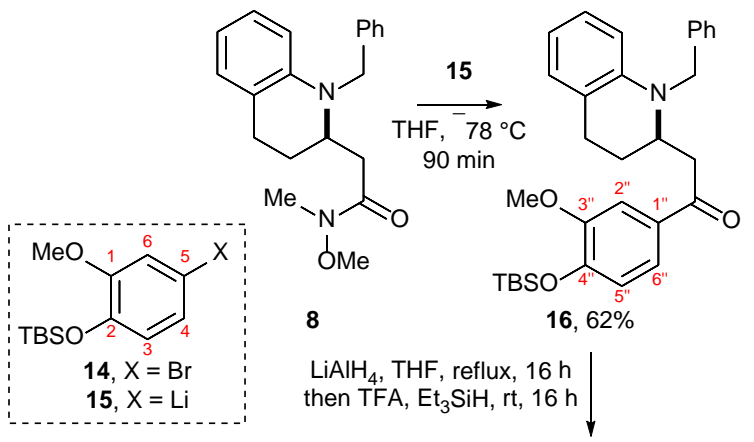

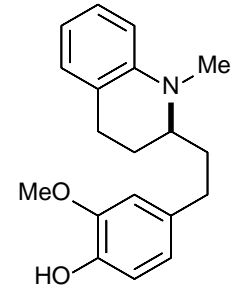

$18,70 \%$

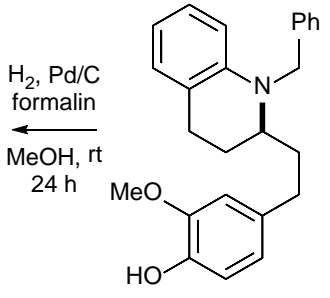

$17,62 \%$
TBS = tert-butyldimethylsilyl.

Comparison of the ${ }^{1} \mathrm{H}$ and ${ }^{13} \mathrm{C}$ NMR data of 18 and (-)galipeine showed excellent agreement, with the spectra being effectively superimposable $\left(\Delta \delta_{\mathrm{H}} \leq 0.04 \mathrm{ppm}, \Delta \delta_{\mathrm{C}} \leq 0.2\right.$ $\mathrm{ppm}) .{ }^{22}$ These data therefore validate our proposal that $\mathbf{1 8}$ and (-)-galipeine are the same (Figure 4). We surmise that the structural misassignment of Jacquemond-Collet et al. arose due to misinterpretation of a ${ }^{3} J_{\mathrm{C}-4^{\prime}-\mathrm{H}-2^{\prime}} \mathrm{HMBC}$ correlation (observed for 18) as ${ }^{2} J_{\mathrm{C}-4^{\prime}-\mathrm{H}-5^{\prime}}$ in the data of the natural product, ${ }^{1}$ as well as a ${ }^{3} J_{\mathrm{C}-3^{\prime}-\mathrm{H}-5^{\prime}}$ HMBC correlation (observed for 18) as ${ }^{2} J_{\mathrm{C}-}$ $3^{\prime}-\mathrm{H}-2^{\prime}$ in the data of natural product, ${ }^{1}$ as this has the result of transposing the positions of the hydroxy and methoxy substituents. (-)-Galipeine was reported to have $[\alpha]_{\mathrm{D}}-13.6^{1}$ although the conditions under which this value was measured, i.e., temperature, concentration and solvent, were not specified. ${ }^{24}$ We investigated the effect of concentration and solvent 
on the observed value of the specific rotation of $\mathbf{1 8}$ and obtained the following data: $[\alpha]_{\mathrm{D}}{ }^{25}-22.0\left(c 0.2\right.$ in $\left.\mathrm{CHCl}_{3}\right) ;[\alpha]_{\mathrm{D}}{ }^{25}$ -22.4 (c 1.0 in $\mathrm{CHCl}_{3}$ ); $[\alpha]_{\mathrm{D}}^{25}-14.0$ (c 1.0 in $\mathrm{MeOH}$ ); $[\alpha]_{\mathrm{D}}^{25}$ -11.9 ( $c$ 1.0 in EtOH). Given the uniform negative value observed in these common solvents, it seems certain that (-)galipeine possesses the absolute $(S)$-configuration. On the basis of all of our data, therefore, the structure of $(-)$-galipeine should henceforth be revised to $(S)-N(1)$-methyl-2-[2'-(3"methoxy-4"-hydroxyphenyl)ethyl]-1,2,3,4-tetrahydroquinoline 18.

\begin{tabular}{|c|c|}
\hline \multicolumn{2}{|c|}{${ }^{1} \mathrm{H}$ NMR $\left(400 \mathrm{MHz} \mathrm{CDCl}_{3}\right)$} \\
\hline 18 & ( )-galipeine \\
\hline $1.73(1 \mathrm{H}, \mathrm{m})$ & $1.70(1 \mathrm{H}, \mathrm{m})^{\mathrm{a}}$ \\
\hline $1.93(3 \mathrm{H}, \mathrm{m})$ & $1.90(3 \mathrm{H}, \mathrm{m})^{\mathrm{a}}$ \\
\hline $2.52(1 \mathrm{H}, \mathrm{ddd})$ & $2.50(1 \mathrm{H}, \mathrm{m})^{\mathrm{a}}$ \\
\hline $2.68(2 \mathrm{H}, \mathrm{m})$ & $2.68(2 \mathrm{H}, \mathrm{m})^{\mathrm{a}}$ \\
\hline $2.86(1 \mathrm{H}, \mathrm{ddd})$ & $2.82(1 \mathrm{H}, \mathrm{m})$ \\
\hline $2.92(3 \mathrm{H}, \mathrm{s})$ & $2.89(3 \mathrm{H}, \mathrm{s})$ \\
\hline $3.30(1 \mathrm{H}, \mathrm{m})$ & $3.26(1 \mathrm{H}, \mathrm{m})$ \\
\hline $3.88(3 \mathrm{H}, \mathrm{s})$ & $3.85(3 \mathrm{H}, \mathrm{s})$ \\
\hline $5.47(1 \mathrm{H}, \mathrm{s})$ & $5.45(1 \mathrm{H}, \mathrm{s})^{\mathrm{a}}$ \\
\hline $6.54(1 \mathrm{H}, \mathrm{d})$ & $6.51(1 \mathrm{H}, \mathrm{d})$ \\
\hline $6.60(1 \mathrm{H}, \mathrm{td})$ & $6.57(1 \mathrm{H}, \mathrm{td})$ \\
\hline $6.69(2 \mathrm{H}, \mathrm{m})$ & $6.65(2 \mathrm{H}, \mathrm{m})$ \\
\hline $6.84(1 \mathrm{H}, \mathrm{d})$ & $6.80(1 \mathrm{H}, \mathrm{d})$ \\
\hline $6.99(1 \mathrm{H}, \mathrm{d})$ & $6.96(1 \mathrm{H}, \mathrm{d})$ \\
\hline $7.10(1 \mathrm{H}, \mathrm{m})$ & $7.06(1 \mathrm{H}, \mathrm{t})$ \\
\hline
\end{tabular}<smiles>CCc1ccc(O)c(OC)c1</smiles>

\begin{tabular}{c|c}
\hline $\mathbf{1 8}$ & ( )-galipeine \\
\hline 23.5 & $23.6(0.1)$ \\
24.3 & $24.4(0.1)$ \\
32.0 & $32.1(0.1)$ \\
33.1 & $33.2(0.1)$ \\
38.1 & $38.2(0.1)$ \\
55.9 & $56.0(0.1)$ \\
58.3 & $58.5(0.2)$ \\
110.5 & $110.7(0.2)$ \\
110.7 & $110.8(0.1)$ \\
114.2 & $114.3(0.1)$ \\
115.3 & $115.4(0.1)$ \\
120.7 & $120.9(0.2)$ \\
121.6 & $121.8(0.2)$ \\
127.1 & $127.2(0.1)$ \\
128.6 & $128.8(0.2)$ \\
133.9 & $134.0(0.1)$ \\
143.6 & $143.7(0.1)$ \\
\hline 145.2 & $145.4(0.2)$ \\
146.3 & $146.5(0.2)$ \\
\hline
\end{tabular}

Figure 4. ${ }^{1} \mathrm{H}$ and ${ }^{13} \mathrm{C}$ NMR data of 18 and (-)-galipeine. Midpoints of all multiplets are quoted. Values of $\Delta \delta_{\mathrm{C}}$ are given in parentheses. ${ }^{\text {TThese }}{ }^{1} \mathrm{H}$ NMR data of (-)-galipeine are corrected here (compared to those reported in Ref 1 ) by analysis of the ${ }^{1} \mathrm{H}$ NMR spectrum of the natural product.

In conclusion, we have prepared authentic samples of the regioisomers (S)-N(1)-methyl-2-[2'-(3"-hydroxy-4"methoxyphenyl)ethyl]-1,2,3,4-tetrahydroquinoline and $(S)$ N(1)-methyl-2-[2'-(3"-methoxy-4"-hydroxyphenyl)ethyl]-

1,2,3,4-tetrahydroquinoline. Comparison of their ${ }^{1} \mathrm{H}$ and ${ }^{13} \mathrm{C}$ NMR data with those reported for (-)-galipeine clearly shows that the data of (S)-N(1)-methyl-2-[2'-(3"-methoxy-4"hydroxyphenyl)ethyl]-1,2,3,4-tetrahydroquinoline match those of the natural product, i.e., the structure of (-)-galipeine was misassigned as N(1)-methyl-2-[2'-(3"-hydroxy-4"methoxyphenyl)ethyl]-1,2,3,4-tetrahydroquinoline in the original isolation study, and this error has been perpetuated in two subsequent synthetic investigations. Comparison of specific rotation data allowed us to assign the absolute (S)configuration for the natural product. The structure of (-)galipeine is thus herein revised to that of $(S)-N(1)$-methyl-2[2'-(3"-methoxy-4"-hydroxyphenyl)ethyl]-1,2,3,4-

tetrahydroquinoline. This study constitutes the unambiguous structural determination of (-)-galipeine, its first total asymmetric synthesis, and assignment of its absolute (S)configuration.

\section{EXPERIMENTAL SECTION}

General Experimental Details. Reactions involving moisturesensitive reagents were carried out under a nitrogen atmosphere using

standard vacuum line techniques and glassware that was flame-dried and cooled under nitrogen before use. Solvents were dried according to the procedure outlined by Grubbs and co-workers. ${ }^{25}$ Water was purified by an Elix ${ }^{\circledR}$ UV-10 system. $n$-BuLi was purchased as a 2.5 $\mathrm{M}$ solution in hexanes and titrated against diphenylacetic acid before use. Organic layers were dried over $\mathrm{MgSO}_{4}$ unless otherwise stated. Thin layer chromatography was performed on aluminium plates coated with $60 \mathrm{~F}_{254}$ silica. Flash column chromatography was performed on Kieselgel 60 silica on a glass column.

Melting points were recorded on a Gallenkamp Hot Stage apparatus and are uncorrected. Optical rotations were recorded on a Perkin-Elmer 241 polarimeter with a water-jacketed $10 \mathrm{~cm}$ cell. Specific rotations are reported in $10^{-1} \mathrm{deg} \mathrm{cm}^{2} \mathrm{~g}^{-1}$ and concentrations in g/100 $\mathrm{mL}$. IR spectra were recorded on a Bruker Tensor 27 FT-IR spectrometer using an ATR module. Selected characteristic peaks are reported in $\mathrm{cm}^{-1}$. NMR spectra were recorded on Bruker Avance spectrometers in the deuterated solvent stated. The field was locked by external referencing to the relevant deuteron resonance. ${ }^{1} \mathrm{H}-{ }^{1} \mathrm{H}$ COSY and ${ }^{1} \mathrm{H}-{ }^{13} \mathrm{C}$ HMQC analyses were used to establish atom connectivity. Low-resolution mass spectra were recorded on either a VG MassLab 20-250 or a Micromass Platform 1 spectrometer. Accurate mass measurements were run on either a Bruker MicroTOF internally calibrated with polyalanine, or a Micromass GCT instrument fitted with a Scientific Glass Instruments BPX5 column $(15 \mathrm{~m} \times 0.25 \mathrm{~mm})$ using amyl acetate as a lock mass.

3-(2'-Bromophenyl)propan-1-ol 2. $\mathrm{NaBH}_{4}$ (225 mg, $6.74 \mathrm{mmol}$ ) was added portionwise to a stirred solution of $\mathbf{1}(771 \mathrm{mg}, 3.37 \mathrm{mmol})$ in THF $(7 \mathrm{~mL})$ at $0{ }^{\circ} \mathrm{C}$, then $\mathrm{BF}_{3} \cdot \mathrm{Et}_{2} \mathrm{O}(0.84 \mathrm{~mL}, 6.7 \mathrm{mmol})$ was added dropwise. The resultant mixture was stirred at $0{ }^{\circ} \mathrm{C}$ for $1 \mathrm{~h}$, then $\mathrm{MeOH}(4 \mathrm{~mL})$ and $1 \mathrm{M}$ aq $\mathrm{HCl}(4 \mathrm{~mL})$ were added sequentially. The resultant mixture was extracted with EtOAc $(3 \times 10 \mathrm{~mL})$ and the combined organic extracts were dried and concentrated in vacuo. Purification via flash column chromatography (eluent $30-40{ }^{\circ} \mathrm{C}$ pet$\left.\mathrm{rol} / \mathrm{Et}_{2} \mathrm{O} / \mathrm{NH}_{4} \mathrm{OH}, 50: 50: 1\right)$ gave 2 as a colourless oil (664 mg, $\left.92 \%\right) ;{ }^{26}$ $\delta_{\mathrm{H}}\left(400 \mathrm{MHz}, \mathrm{CDCl}_{3}\right) 1.37(1 \mathrm{H}, \mathrm{t}, J 5.8, \mathrm{OH}), 1.87-1.96(2 \mathrm{H}, \mathrm{m}$, $\left.\mathrm{C}(2) \mathrm{H}_{2}\right), 2.85$ (2H, t, $J$ 7.8, C(3) $\left.\mathrm{H}_{2}\right), 3.72$ (2H, app q, $J$ 5.8, C(1) $\mathrm{H}_{2}$ ), 7.04-7.11 (1H, m, C(4')H), 7.23-7.29 (2H, m, C(5')H, C(6')H), 7.55 (1H, d, $J$ 8.1, C $\left.\left(3^{\prime}\right) H\right)$.

(E)-5-(2'-Bromophenyl)- $N$-methoxy- $N$-methylpent-2-enamide 4. DMSO (0.53 mL, $7.4 \mathrm{mmol})$ was added dropwise to a stirred solution of $(\mathrm{COCl})_{2}(0.32 \mathrm{~mL}, 3.7 \mathrm{mmol})$ in $\mathrm{CH}_{2} \mathrm{Cl}_{2}(10 \mathrm{~mL})$ at $-78{ }^{\circ} \mathrm{C}$ and the resultant solution was stirred at $-78{ }^{\circ} \mathrm{C}$ for $20 \mathrm{~min}$. A solution of 2 (400 mg, $1.86 \mathrm{mmol})$ in $\mathrm{CH}_{2} \mathrm{Cl}_{2}(1 \mathrm{~mL})$ was then added and the resultant solution was stirred at $-78{ }^{\circ} \mathrm{C}$ for $40 \mathrm{~min}$. $\mathrm{Et}_{3} \mathrm{~N}(1.7 \mathrm{~mL}, 11$ $\mathrm{mmol}$ ) was then added, the resultant solution was allowed to warm to rt and 3 (1.01 g, $2.79 \mathrm{mmol}$ ) was added portionwise. The resultant solution was stirred at rt for $16 \mathrm{~h}$. Satd aq $\mathrm{K}_{2} \mathrm{CO}_{3}(20 \mathrm{~mL})$ was added and the aqueous layer was extracted with $\mathrm{CH}_{2} \mathrm{Cl}_{2}(3 \times 20 \mathrm{~mL})$. The combined organics were washed with brine $(60 \mathrm{~mL})$, then dried and concentrated in vacuo. Purification via flash column chromatography (eluent $30-40{ }^{\circ} \mathrm{C}$ petrol $/ \mathrm{Et}_{2} \mathrm{O} / \mathrm{NH}_{4} \mathrm{OH}, 50: 50: 1$ ) gave 4 as a pale yellow oil (435 mg, 78\%, >95:5 dr [(E):(Z) ratio]); $v_{\max } 2935,1663$, $1632 ; \delta_{\mathrm{H}}\left(400 \mathrm{MHz}, \mathrm{CDCl}_{3}\right) 2.61\left(2 \mathrm{H}\right.$, app q, $\left.J 7.5, \mathrm{C}(4) \mathrm{H}_{2}\right), 2.95$ ( $2 \mathrm{H}, \mathrm{t}, J$ 7.5, C(5) $\left.\mathrm{H}_{2}\right), 3.28$ (3H, s, NMe), 3.70 (3H, s, OMe), 6.54 (1H, d, J 15.4, C(2)H), 7.02-7.14 (2H, m, C(3)H, C(4')H), 7.23-7.32 $\left(2 \mathrm{H}, \mathrm{m}, \mathrm{C}\left(5^{\prime}\right) H, \mathrm{C}\left(6^{\prime}\right) H\right), 7.58\left(1 \mathrm{H}, \mathrm{d}, J\right.$ 7.9, C(3')H); $\delta_{\mathrm{C}}(100 \mathrm{MHz}$, $\left.\mathrm{CDCl}_{3}\right) 32.3$ (C(4)), 32.5 (NMe), 34.9 (C(5)), 61.6 (OMe), 119.5 (C(2)), $124.3\left(C\left(2^{\prime}\right)\right), 127.5\left(C\left(5^{\prime}\right)\right), 127.8\left(C\left(4^{\prime}\right)\right), 130.4\left(C\left(6^{\prime}\right)\right), 132.8$ (C(3')), $140.2\left(C\left(1^{\prime}\right)\right), 146.0(C(3)), 166.7(C(1)) ; \mathrm{m} / \mathrm{z}\left(\mathrm{ESI}^{+}\right) 300$ $\left(\left[\mathrm{M}\left({ }^{81} \mathrm{Br}\right)+\mathrm{H}\right]^{+}, 100 \%\right), 298\left(\left[\mathrm{M}\left({ }^{79} \mathrm{Br}\right)+\mathrm{H}\right]^{+}, 100 \%\right) ; \mathrm{HRMS}\left(\mathrm{ESI}^{+}\right)$ $\mathrm{C}_{13} \mathrm{H}_{17}{ }^{81} \mathrm{BrNO}_{2}{ }^{+}\left(\left[\mathrm{M}\left({ }^{81} \mathrm{Br}\right)+\mathrm{H}\right]^{+}\right)$requires 300.0417; found 300.0414; $\mathrm{C}_{13} \mathrm{H}_{17}{ }^{79} \mathrm{BrNO}_{2}{ }^{+}\left(\left[\mathrm{M}\left({ }^{79} \mathrm{Br}\right)+\mathrm{H}\right]^{+}\right)$requires 298.0437; found 298.0435 .

$(R, R)-3-[N$-Benzyl- $N$-( $\alpha$-methyl-p-methoxybenzyl)amino]-5-(2'bromophenyl)- $\boldsymbol{N}$-methoxy- $\boldsymbol{N}$-methylpentanamide 6 . $n$-BuLi $(2.3 \mathrm{M}$ in hexanes, $0.45 \mathrm{~mL}, 1.0 \mathrm{mmol}$ ) was added dropwise to a stirred solution of $(R)$ - $N$-benzyl- $N$-( $\alpha$-methyl- $p$-methoxybenzyl)amide (259 mg, $1.07 \mathrm{mmol},>98 \%$ ee) in THF ( $2 \mathrm{~mL})$ at $-78{ }^{\circ} \mathrm{C}$ and the resultant solution was stirred at $-78{ }^{\circ} \mathrm{C}$ for $30 \mathrm{~min}$. A solution of $4(200 \mathrm{mg}, 0.671$ mmol, $>95: 5 \mathrm{dr}\left[(E):(Z)\right.$ ratio]) in THF $(1 \mathrm{~mL})$ at $-78{ }^{\circ} \mathrm{C}$ was then added and the resultant solution was stirred at $-78{ }^{\circ} \mathrm{C}$ for $2 \mathrm{~h}$. Satd aq $\mathrm{NH}_{4} \mathrm{Cl}(1 \mathrm{~mL})$ was then added and the resultant mixture was allowed 
to warm to rt, then concentrated in vacuo. The residue was partitioned between $\mathrm{CH}_{2} \mathrm{Cl}_{2}(10 \mathrm{~mL})$ and $10 \%$ aq citric acid $(10 \mathrm{~mL})$, and the organic layer was washed sequentially with satd aq $\mathrm{NaHCO}_{3}(10 \mathrm{~mL})$ and brine $(10 \mathrm{~mL})$, then dried and concentrated in vacuo to give 6 in $>95: 5 \mathrm{dr}$. Purification via flash column chromatography (eluent 30-40 ${ }^{\circ} \mathrm{C}$ petrol/Et $t_{2} \mathrm{O} / \mathrm{NH}_{4} \mathrm{OH}, 50: 50: 1$ ) gave 6 as a pale yellow oil (273 mg, 80\%, >95:5 dr); $[\alpha]_{\mathrm{D}}{ }^{25}+21.8$ (c 1.0 in $\mathrm{CHCl}_{3}$ ); $v_{\max }$ 2998, 2981, 2969, 2932, 2905, 2886, 2868, 1659; $\delta$ н $\left(400 \mathrm{MHz}, \mathrm{CDCl}_{3}\right) 1.39$ (3H, d, $J$ 7.0, $\mathrm{C}(\alpha) M e), 1.55-1.66\left(1 \mathrm{H}, \mathrm{m}, \mathrm{C}(4) H_{\mathrm{A}}\right), 1.73-1.84(1 \mathrm{H}, \mathrm{m}$, $\left.\mathrm{C}(4) H_{\mathrm{B}}\right), 2.00\left(1 \mathrm{H}, \mathrm{d}, J 14.0, \mathrm{C}(2) H_{\mathrm{A}}\right), 2.21-2.31\left(1 \mathrm{H}, \mathrm{m}, \mathrm{C}(2) H_{\mathrm{B}}\right)$, 2.70 (1H, ddd, $J$ 13.8, 11.8, 5.0, C(5) $\left.H_{\mathrm{A}}\right), 3.07$ (3H, s, NMe), 3.19 (1H, ddd, $J$ 13.8, 11.6, 4.9, C(5)Hв), 3.43 (3H, s, NOMe), 3.59-3.66 $(1 \mathrm{H}, \mathrm{m}, \mathrm{C}(3) \mathrm{H}), 3.60\left(1 \mathrm{H}, \mathrm{d}, J\right.$ 14.8, $\left.\mathrm{NCH}_{\mathrm{A}} \mathrm{H}_{\mathrm{B}} \mathrm{Ph}\right), 3.79(3 \mathrm{H}, \mathrm{s}$, ArOMe), $3.87(1 \mathrm{H}, \mathrm{q}, J 7.0, \mathrm{C}(\alpha) H), 3.93\left(1 \mathrm{H}, \mathrm{d}, J 14.8, \mathrm{NCH}_{\mathrm{A}} H_{\mathrm{B}} \mathrm{Ph}\right)$, 6.85 (2H, d, J 8.6, C(3")H, C(5")H), 6.99-7.05 (1H, m, C(4')H), 7.167.28 (5H, m, C(5')H, C(6')H, C(2")H, C(6")H, Ph), 7.36 (2H, t, J 7.5, $P h), 7.50(1 \mathrm{H}, \mathrm{d}, J$ 7.8, C(3')H), 7.54 (2H, d, $J 7.5, P h) ; \delta \mathrm{c}(100 \mathrm{MHz}$, $\left.\mathrm{CDCl}_{3}\right) 20.0(\mathrm{C}(\alpha) \mathrm{Me}), 32.1(\mathrm{NMe}), 33.8(C(2)), 33.9(C(4)), 34.2$ $(C(5)), 50.2\left(\mathrm{NCH}_{2} \mathrm{Ph}\right), 52.9(C(3)), 55.2$ (NOMe), $56.6(C(\alpha)), 60.8$ (ArOMe), $113.4\left(C\left(3^{\prime \prime}\right), C\left(5^{\prime \prime}\right)\right), 124.4\left(C\left(2^{\prime}\right)\right), 126.6(p-P h), 127.2$ (C(4')), $127.4\left(C\left(5^{\prime}\right)\right), 128.3,128.3$ (o,m-Ph), 129.0 (C(2"), $\left.C\left(6^{\prime \prime}\right)\right)$, $130.2\left(C\left(6^{\prime}\right)\right), 132.6\left(C\left(3^{\prime}\right)\right), 135.1\left(C\left(1^{\prime \prime}\right)\right), 141.5(i-P h), 142.1\left(C\left(1^{\prime}\right)\right)$, $158.5\left(C\left(4^{4}\right)\right), 173.4(C(1)) ; \mathrm{m} / \mathrm{z}\left(\mathrm{ESI}^{+}\right) 541\left(\left[\mathrm{M}\left({ }^{81} \mathrm{Br}\right)+\mathrm{H}\right]^{+}, 100 \%\right)$, $539\left(\left[\mathrm{M}\left({ }^{79} \mathrm{Br}\right)+\mathrm{H}\right]^{+}, \quad 100 \%\right) ; \quad$ HRMS $\quad\left(\mathrm{ESI}^{+}\right) \quad \mathrm{C}_{29} \mathrm{H}_{36}{ }^{81} \mathrm{BrN}_{2} \mathrm{O}_{3}{ }^{+}$ $\left(\left[\mathrm{M}\left({ }^{81} \mathrm{Br}\right)+\mathrm{H}\right]^{+}\right)$requires 541.1883; found 541.1882; $\mathrm{C}_{29} \mathrm{H}_{36}{ }^{79} \mathrm{BrN}_{2} \mathrm{O}_{3}{ }^{+}$ $\left(\left[\mathrm{M}\left({ }^{79} \mathrm{Br}\right)+\mathrm{H}\right]^{+}\right)$requires 539.1904; found 539.1902.

(R)-3-( $N$-Benzylamino)-5-(2'-bromophenyl)- $N$-methoxy- $N$ -

methylpentanamide 7. $\mathrm{Et}_{3} \mathrm{SiH}(25 \mu \mathrm{L}, 0.16 \mathrm{mmol})$ was added to a stirred solution of $6(87 \mathrm{mg}, 1.10 \mathrm{mmol},>95: 5 \mathrm{dr})$ in $\mathrm{HCO}_{2} \mathrm{H}(0.6$ $\mathrm{mL}$ ) at $\mathrm{rt}$ and the resultant solution was heated at $90^{\circ} \mathrm{C}$ for $16 \mathrm{~h}$, then allowed to cool to rt and concentrated in vacuo. The residue was partitioned between $\mathrm{CH}_{2} \mathrm{Cl}_{2}(5 \mathrm{~mL})$ and satd aq $\mathrm{NaHCO}_{3}(5 \mathrm{~mL})$ and the organic layer was washed with brine $(5 \mathrm{~mL})$, then dried and concentrated in vacuo. Purification via flash column chromatography (eluent 30-40 ${ }^{\circ} \mathrm{C}$ petrol/ $/ \mathrm{Et}_{2} \mathrm{O} / \mathrm{NH}_{4} \mathrm{OH}, 50: 50: 1$ ) gave 7 as a colourless oil (35 mg, 81\%); $[\alpha]_{\mathrm{D}}^{25}-7.1$ (c 1.0 in $\mathrm{CHCl}_{3}$ ); $v_{\max } 3324,3085,3027,2965$, $2935,2861,1658 ; \delta_{\mathrm{H}}\left(400 \mathrm{MHz}, \mathrm{CDCl}_{3}\right) 1.79-1.89\left(2 \mathrm{H}, \mathrm{m}, \mathrm{C}(4) \mathrm{H}_{2}\right)$, 2.67 (2H, app d, $J$ 5.8, C(2) $H_{2}$ ), 2.80-2.86 (2H, m, C(5) $\left.H_{2}\right)$, 3.16-3.23 $(1 \mathrm{H}, \mathrm{m}, \mathrm{C}(3) \mathrm{H}), 3.18$ (3H, s, NMe), 3.67 (3H, s, OMe), $3.81(1 \mathrm{H}, \mathrm{d}, J$ 12.9, $\left.\mathrm{NCH}_{\mathrm{A}} \mathrm{H}_{\mathrm{B}} \mathrm{Ph}\right), 3.85\left(1 \mathrm{H}, \mathrm{d}, J 12.9, \mathrm{NCH}_{\mathrm{A}} \mathrm{H}_{\mathrm{B}} \mathrm{Ph}\right), 7.01-7.08(1 \mathrm{H}$, m, C(4')H), 7.20-7.39 (7H, m, C(5')H, C(6')H, Ph), 7.52 (1H, d, J 7.8, $\left.\mathrm{C}\left(3^{\prime}\right) H\right) ; \delta_{\mathrm{C}}\left(100 \mathrm{MHz}, \mathrm{CDCl}_{3}\right) 32.0(C(3)), 32.4(C(5)), 34.3(C(4))$, $36.4(\mathrm{C}(2))$, $51.0\left(\mathrm{NCH}_{2} \mathrm{Ph}\right), 53.8(\mathrm{NMe}), 61.2(\mathrm{OMe}), 124.4\left(\mathrm{C}\left(2^{\prime}\right)\right)$, 126.8, 127.5, 127.5 (C(4'), $\left.C\left(5^{\prime}\right), p-P h\right), 128.2,128.3(o, m-P h), 130.3$ (C(6')), $132.7\left(C\left(3^{\prime}\right)\right), 140.6(i-P h), 141.6\left(C\left(1^{\prime}\right)\right), 173.3(C(1)) ; \mathrm{m} / \mathrm{z}$ $\left(\mathrm{ESI}^{+}\right) 407\left(\left[\mathrm{M}\left({ }^{81} \mathrm{Br}\right)+\mathrm{H}\right]^{+}, 100 \%\right), 405\left(\left[\mathrm{M}\left({ }^{79} \mathrm{Br}\right)+\mathrm{H}\right]^{+}, 100 \%\right)$; HRMS $\left(\mathrm{ESI}^{+}\right) \mathrm{C}_{20} \mathrm{H}_{26}{ }^{81} \mathrm{BrN}_{2} \mathrm{O}_{2}{ }^{+}\left(\left[\mathrm{M}\left({ }^{81} \mathrm{Br}\right)+\mathrm{H}\right]^{+}\right)$requires 407.1152; found 407.1148; $\mathrm{C}_{20} \mathrm{H}_{26}{ }^{79} \mathrm{BrN}_{2} \mathrm{O}_{2}{ }^{+}\left(\left[\mathrm{M}\left({ }^{79} \mathrm{Br}\right)+\mathrm{H}\right]^{+}\right)$requires 405.1172; found 405.1169.

(R)-2-[N(1')-Benzyl-1',2',3',4'-tetrahydroquinolin-2'-yl]- $N$ -

methoxy- $N$-methylacetamide 8 . $\mathrm{Pd}(\mathrm{OAc})_{2}(26 \mathrm{mg}, 0.12 \mathrm{mmol})$ was added to a stirred suspension of 7 (943 $\mathrm{mg}, 2.33 \mathrm{mmol}$ ), XPhos (163 mg, $0.349 \mathrm{mmol})$ and $\mathrm{Cs}_{2} \mathrm{CO}_{3}(1.51 \mathrm{~g}, 4.65 \mathrm{mmol})$ in PhMe $(30 \mathrm{~mL})$ and the resultant solution was heated at reflux for $24 \mathrm{~h}$, then allowed to cool to rt and concentrated in vacuo. The residue was partitioned between $\mathrm{CH}_{2} \mathrm{Cl}_{2}(100 \mathrm{~mL})$ and $\mathrm{H}_{2} \mathrm{O}(100 \mathrm{~mL})$ and the organic layer was dried and concentrated in vacuo. Purification via flash column chromatography (eluent $30-40{ }^{\circ} \mathrm{C}$ petrol/ $\mathrm{Et}_{2} \mathrm{O} / \mathrm{NH}_{4} \mathrm{OH}, 50: 50: 1$ ) gave 8 as a pale yellow solid (751 mg, quant); mp 58-60 ${ }^{\circ} \mathrm{C}$; $[\alpha]_{\mathrm{D}}{ }^{25}-11.0$ (c 1.0 in $\left.\mathrm{CHCl}_{3}\right) ; v_{\max } 3027,2968,2935,2865,1658 ; \delta_{\mathrm{H}}(400 \mathrm{MHz}$, $\left.\mathrm{CDCl}_{3}\right)$ 1.97-2.13 (2H, m, C(3') $\left.H_{2}\right), 2.71$ (2H, app d, $J$ 6.5, C(2)H $H_{2}$, $2.78\left(1 \mathrm{H}, \mathrm{dt}, J\right.$ 16.5, 3.7, C(4') $\left.H_{\mathrm{A}}\right), 2.97$ (1H, ddd, $J$ 16.5, 12.8, 5.7, $\left.\mathrm{C}\left(4^{\prime}\right) \mathrm{H}_{\mathrm{B}}\right), 3.16(3 \mathrm{H}, \mathrm{s}, \mathrm{NMe}), 3.58$ (3H, s, OMe), 4.04-4.10 (1H, m, $\left.\mathrm{C}\left(2^{\prime}\right) H\right), 4.53\left(1 \mathrm{H}, \mathrm{d}, J\right.$ 17.2, $\left.\mathrm{NCH}_{\mathrm{A}} \mathrm{H}_{\mathrm{B}} \mathrm{Ph}\right), 4.59(1 \mathrm{H}, \mathrm{d}, J$ 17.2, $\left.\mathrm{NCH}_{\mathrm{A}} H_{\mathrm{B}} \mathrm{Ph}\right), 6.45\left(1 \mathrm{H}, \mathrm{d}, J 7.7, \mathrm{C}\left(8^{\prime}\right) H\right), 6.60\left(1 \mathrm{H}, \mathrm{t}, J 7.7, \mathrm{C}\left(6^{\prime}\right) H\right)$, $6.97(1 \mathrm{H}, \mathrm{t}, J$ 7.7, C(7')H), 7.04 (1H, d, $J$ 7.7, C(5')H), 7.19-7.33 (5H, $\mathrm{m}, \mathrm{Ph}) ; \delta_{\mathrm{C}}\left(100 \mathrm{MHz}, \mathrm{CDCl}_{3}\right) 23.6\left(C\left(4^{\prime}\right)\right), 25.3\left(C\left(3^{\prime}\right)\right), 32.0(\mathrm{NMe})$, $34.9(C(2))$, $53.9\left(\mathrm{NCH}_{2} \mathrm{Ph}\right), 54.8\left(C\left(2^{\prime}\right)\right), 61.3(\mathrm{OMe}), 111.7\left(C\left(8^{\prime}\right)\right)$, 115.9 (C(6')), 121.2 (C(4'a)), $126.4(o-P h), 126.7(p-P h), 127.1$ $\left(C\left(7^{\prime}\right)\right), 128.5(m-P h), 129.1\left(C\left(5^{\prime}\right)\right), 139.0(i-P h), 144.0\left(C\left(8^{\prime} a\right)\right)$,
$172.5(C(1)) ; m / z\left(\mathrm{ESI}^{+}\right) 325\left([\mathrm{M}+\mathrm{H}]^{+}, 100 \%\right) ; \mathrm{HRMS}\left(\mathrm{ESI}^{+}\right)$ $\mathrm{C}_{20} \mathrm{H}_{25} \mathrm{~N}_{2} \mathrm{O}_{2}{ }^{+}\left([\mathrm{M}+\mathrm{H}]^{+}\right)$requires 325.1911; found 325.1909.

(R)-N(1)-Benzyl-2-[2'-Oxo-2'-(3"'-tert-butyldimethylsilyloxy-4"'methoxyphenyl)ethyl]-1,2,3,4-tetrahydroquinoline 11 . Step 1 . Preparation of O-tert-butyldimethylsilyl-5-bromoguaiacol 9. tertButyldimethylsilyl chloride $(1.50 \mathrm{~g}, 10.0 \mathrm{mmol})$ was added to a stirred solution of 5-bromoguaiacol (2.00 g, $9.85 \mathrm{mmol})$ and imidazole (1.36 g, $20.0 \mathrm{mmol})$ in DMF (5 mL) at rt and the resultant solution was stirred at rt for $16 \mathrm{~h} . \mathrm{H}_{2} \mathrm{O}(15 \mathrm{~mL})$ was then added and the resultant mixture was extracted with hexane $(5 \times 15 \mathrm{~mL})$. The combined organics were dried and concentrated in vacuo to give $\mathbf{9}$ as a colourless oil (2.92 g, 93\%); ${ }^{27} \delta_{\mathrm{H}}\left(400 \mathrm{MHz}, \mathrm{CDCl}_{3}\right) 0.15\left(6 \mathrm{H}, \mathrm{s}, \mathrm{Si}_{2} e_{2}\right.$ ), 0.99 (9H, s, SiCMe $), 3.78$ (3H, s, OMe), $6.71(1 \mathrm{H}, \mathrm{d}, J$ 8.6, C(3)H), 6.98 (1H, d, J 2.4, C(6)H), 7.02 (1H, dd, J 8.6, 2.4, C(4)H).

Step 2. $n$-BuLi (2.3 M in hexanes, $1.9 \mathrm{~mL}, 4.3 \mathrm{mmol}$ ) was added dropwise to a stirred solution of 9 (1.37 g, $4.32 \mathrm{mmol})$ in THF (15 $\mathrm{mL})$ at $-78{ }^{\circ} \mathrm{C}$ and the resultant solution was stirred at $-78{ }^{\circ} \mathrm{C}$ for 30 min. A solution of $8(200 \mathrm{mg}, 0.616 \mathrm{mmol})$ in THF $(2 \mathrm{~mL})$ at $-78{ }^{\circ} \mathrm{C}$ was then added and the resultant solution was stirred at $-78{ }^{\circ} \mathrm{C}$ for 90 min. Satd aq $\mathrm{NH}_{4} \mathrm{Cl}(3 \mathrm{~mL})$ was then added and the resultant mixture was allowed to warm to rt and concentrated in vacuo. The residue was partitioned between $\mathrm{CH}_{2} \mathrm{Cl}_{2}(20 \mathrm{~mL})$ and $\mathrm{H}_{2} \mathrm{O}(20 \mathrm{~mL})$, and the organic layer was dried and concentrated in vacuo. Purification via flash column chromatography (eluent $30-40{ }^{\circ} \mathrm{C}$ petrol/Et $2 \mathrm{O} / \mathrm{NH}_{4} \mathrm{OH}$, 85:17:1) gave 11 as a yellow oil (273 mg, $88 \%$ ); $[\alpha]_{\mathrm{D}}{ }^{25}-7.7$ (c 1.0 in $\left.\mathrm{CHCl}_{3}\right) ; v_{\max } 2953,2930,2857,1671 ; \delta_{\mathrm{H}}\left(400 \mathrm{MHz}, \mathrm{CDCl}_{3}\right) 0.15$ (6H, s, SiMe $), 0.99$ (9H, s, SiCMe $), 1.97$ (1H, ddt, J 13.1, 5.7, 2.9, $\left.\mathrm{C}(3) H_{\mathrm{A}}\right), 2.09\left(1 \mathrm{H}, \mathrm{tt}, J 13.1,4.8, \mathrm{C}(3) H_{\mathrm{B}}\right), 2.74-2.81(1 \mathrm{H}, \mathrm{m}$, C(4) $\left.H_{\mathrm{A}}\right), 2.96$ (1H, ddd, $J$ 16.7, 13.1, 5.7, C(4)HB $), 3.15$ (2H, d, $J$ 6.7, $\left.\mathrm{C}\left(1^{\prime}\right) H_{2}\right), 3.86$ (3H, s, OMe), 4.15-4.21 (1H, m, C(2)H), 4.49 (1H, d, $J$ 17.2, $\left.\mathrm{NCH}_{\mathrm{A}} \mathrm{H}_{\mathrm{B}} \mathrm{Ph}\right), 4.56$ (1H, d, J 17.2, $\left.\mathrm{NCH}_{\mathrm{A}} \mathrm{H}_{\mathrm{B}} \mathrm{Ph}\right), 6.45$ (1H, app d, $J$ 8.2, C(8)H), $6.62(1 \mathrm{H}, \mathrm{td}, J$ 7.3, 0.9, C(6)H), $6.83(1 \mathrm{H}, \mathrm{d}, J$ 8.5, C(5")H), 6.95-7.00 (1H, m, C(7)H), 7.04 (1H, d, J 7.3, C(5)H), 7.187.31 (5H, m, $P h), 7.43$ (1H, d, $J$ 2.2, C(2")H), 7.49 (1H, dd, $J$ 8.5, 2.2, $\left.\mathrm{C}\left(6^{\prime \prime}\right) H\right) ; \delta_{\mathrm{C}}\left(100 \mathrm{MHz}, \mathrm{CDCl}_{3}\right)-4.6\left(\mathrm{SiMe}_{2}\right), 18.4\left(\mathrm{SiCMe}_{3}\right), 23.6$ (C(4)), 25.4 (C(3)), $25.7\left(\mathrm{SiCMe}_{3}\right), 40.8\left(C\left(1^{\prime}\right)\right), 54.0\left(\mathrm{NCH}_{2} \mathrm{Ph}\right), 54.9$ (C(2)), 55.5 (OMe), $110.8\left(C\left(5^{\prime \prime}\right)\right), 111.8(C(8)), 116.0(C(6)), 120.3$ $\left(C\left(2^{\prime \prime)}\right), 121.2\right.$ (C(4a)), $123.3\left(C\left(6^{\prime \prime}\right)\right), 126.4(o, m-P h), 126.8(p-P h)$, $127.2(C(7)), 128.6(o, m-P h), 129.1(C(5)), 130.5(C(1 ")), 138.9(i-$ Ph), 144.1 (C(8a)), 145.0 (C(3")), 155.5 (C(4")), 197.6 (C(2')); m/z $\left(\mathrm{ESI}^{+}\right) 502\left([\mathrm{M}+\mathrm{H}]^{+}, 100 \%\right) ; \mathrm{HRMS}\left(\mathrm{ESI}^{+}\right) \mathrm{C}_{31} \mathrm{H}_{40} \mathrm{NO}_{3} \mathrm{Si}^{+}\left([\mathrm{M}+\mathrm{H}]^{+}\right)$ requires 502.2772; found 502.2768.

(S)-N(1)-Benzyl-2-[2'-(3"'-hydroxy-4"'-methoxyphenyl)ethyl]1,2,3,4-tetrahydroquinoline 12. Step $1 . \mathrm{LiAlH}_{4}(2.0 \mathrm{M}$ in THF, 0.46 $\mathrm{mL}, 0.91 \mathrm{mmol}$ ) was added dropwise to a stirred solution of 11 (229 $\mathrm{mg}, 0.456 \mathrm{mmol})$ in THF $(3.2 \mathrm{~mL})$ at $0{ }^{\circ} \mathrm{C}$. The resultant solution was heated at reflux for $16 \mathrm{~h}$ and then allowed to cool to rt. $2 \mathrm{M}$ aq $\mathrm{NaOH}$ $(0.5 \mathrm{~mL})$ was then added and the resultant mixture was heated at reflux for $3 \mathrm{~h}$. The resultant mixture was allowed to cool to rt and then concentrated in vacuo. The residue was dissolved in $\mathrm{Et}_{2} \mathrm{O}$ and filtered through a short plug of silica (eluent $\mathrm{Et}_{2} \mathrm{O}$ ), and the filtrate was concentrated in vacuo.

Step $2 . \mathrm{Et}_{3} \mathrm{SiH}(0.73 \mathrm{~mL}, 4.6 \mathrm{mmol})$ was added to a stirred solution of the residue from the previous step in TFA $(2.2 \mathrm{~mL})$ at $\mathrm{rt}$ and the resultant solution was stirred at rt for $16 \mathrm{~h}$, then concentrated in vacuo. The residue was partitioned between $\mathrm{CH}_{2} \mathrm{Cl}_{2}(10 \mathrm{~mL})$ and satd aq $\mathrm{NaHCO}_{3}(10 \mathrm{~mL})$, and the organic layer was dried and concentrated in vacuo. Purification via flash column chromatography (eluent 30-40 ${ }^{\circ} \mathrm{C}$ petrol/ $\left./ \mathrm{Et}_{2} \mathrm{O} / \mathrm{NH}_{4} \mathrm{OH}, 75: 25: 1\right)$ gave 12 as a pale yellow oil $(85 \mathrm{mg}$, $50 \%$ ); $[\alpha]_{\mathrm{D}}{ }^{25}+0.5$ (c 1.0 in $\mathrm{CHCl}_{3}$ ); $v_{\max } 3513,3027,2980,2971$, 2933, 2860, 1600, 1510, 1498; $\delta_{\mathrm{H}}\left(400 \mathrm{MHz}, \mathrm{CDCl}_{3}\right) 1.77-2.09(4 \mathrm{H}$, m, C(3) $\left.H_{2}, \mathrm{C}\left(1^{\prime}\right) H_{2}\right), 2.45$ (1H, ddd, $\left.J 14.0,9.6,6.8, \mathrm{C}\left(2^{\prime}\right) H_{\mathrm{A}}\right), 2.61$ (1H, ddd, $\left.J 14.0,9.6,5.4, \mathrm{C}(2 \mathrm{\prime}) H_{\mathrm{B}}\right), 2.75$ (1H, dt, $\left.J 16.5,3.9, \mathrm{C}(4) H_{\mathrm{A}}\right)$, 2.93 (1H, ddd, $\left.J 16.5,12.3,5.9, \mathrm{C}(4) H_{\mathrm{B}}\right), 3.35-3.42(1 \mathrm{H}, \mathrm{m}, \mathrm{C}(2) H)$, 3.85 (3H, s, OMe), $4.40\left(1 \mathrm{H}, \mathrm{d}, J 17.0, \mathrm{NCH}_{\mathrm{A}} \mathrm{H}_{\mathrm{B}} \mathrm{Ph}\right), 4.56(1 \mathrm{H}, \mathrm{d}, J$ 17.0, $\left.\mathrm{NCH}_{\mathrm{A}} \mathrm{H}_{\mathrm{B}} \mathrm{Ph}\right), 5.54(1 \mathrm{H}, \mathrm{s}, \mathrm{OH}), 6.41(1 \mathrm{H}, \mathrm{d}, J$ 8.0, C(8)H), 6.55-6.62 (2H, m, C(6)H, C(6")H), 6.72-6.75 (2H, m, C(2")H, C(5")H), 6.94 (1H, t, J 8.0, C(7)H), 7.01 (1H, d, J 7.3, C(5)H), 7.19$7.31(5 \mathrm{H}, \mathrm{m}, \mathrm{Ph}) ; \delta_{\mathrm{C}}\left(100 \mathrm{MHz}, \mathrm{CDCl}_{3}\right) 23.6(C(4)), 24.2(C(3)), 31.6$ (C(2')), $33.5\left(C\left(1^{\prime}\right)\right), 54.0\left(\mathrm{NCH}_{2} \mathrm{Ph}\right), 56.0(\mathrm{OMe}), 57.2(C(2)), 110.6$ (C(5")), $111.7(C(8)), 114.4\left(C\left(2^{\prime \prime}\right)\right), 115.5(C(6)), 119.5\left(C\left(6^{\prime \prime}\right)\right)$, 
121.6 (C(4a)), 126.5 (o,m-Ph), $126.6(p-P h), 127.0$ (C(7)), 128.5 (o,m-Ph), 128.9 (C(5)), $135.1\left(C\left(1^{\prime \prime}\right)\right), 139.2(i-P h), 144.5\left(C\left(4^{\prime \prime}\right)\right)$ 144.7 (C(8a)), $145.5\left(C\left(3^{\prime \prime}\right)\right) ; \mathrm{m} / \mathrm{z}\left(\mathrm{ESI}^{+}\right) 374\left([\mathrm{M}+\mathrm{H}]^{+}, 100 \%\right)$; HRMS $\left(\mathrm{ESI}^{+}\right) \mathrm{C}_{25} \mathrm{H}_{28} \mathrm{NO}_{2}{ }^{+}\left([\mathrm{M}+\mathrm{H}]^{+}\right)$requires 374.2115; found 374.2115.

(S)-N(1)-Methyl-2-[2'-(3"'-hydroxy-4"'-methoxyphenyl)ethyl]1,2,3,4-tetrahydroquinoline 13. $\mathrm{Pd} / \mathrm{C}$ ( $27 \mathrm{mg}, 40 \% \mathrm{w} / \mathrm{w}$ of substrate 12) was added to a stirred solution of $12(68 \mathrm{mg}, 0.18 \mathrm{mmol})$ and formalin ( $37 \%$ aq $\mathrm{HCHO}, 0.13 \mathrm{~mL}, 1.8 \mathrm{mmol}$ ) in degassed $\mathrm{MeOH}$ (3 $\mathrm{mL})$ at rt. The resultant suspension was stirred under $\mathrm{H}_{2}(1 \mathrm{~atm})$ at $\mathrm{rt}$ for $24 \mathrm{~h}$. The resultant suspension was filtered through a short plug of Celite $^{\circledR}$ (eluent $\mathrm{MeOH}$ ) and the filtrate was concentrated in vacuo. Purification via flash column chromatography (eluent $30-40{ }^{\circ} \mathrm{C}$ pet$\mathrm{rol} / \mathrm{Et}_{2} \mathrm{O} / \mathrm{NH}_{4} \mathrm{OH}, 66: 33: 1$ ) gave $\mathbf{1 3}$ as a pale orange solid (31 mg, $57 \%) ;{ }^{11,13} \mathrm{mp} 134-138{ }^{\circ} \mathrm{C}$; $[\alpha]_{\mathrm{D}}{ }^{25}-26.2$ (c 1.0 in $\left.\mathrm{CHCl}_{3}\right)$; $v_{\max } 3501$, 2980, 2934, 1601, 1510, 1501; Н $_{\text { }}\left(400 \mathrm{MHz}, \mathrm{CDCl}_{3}\right) 1.66-1.76(1 \mathrm{H}$, m, C $\left.\left(1^{\prime}\right) H_{\mathrm{A}}\right), 1.84-1.99\left(3 \mathrm{H}, \mathrm{m}, \mathrm{C}(3) H_{2}, \mathrm{C}\left(1^{\prime}\right) H_{\mathrm{B}}\right), 2.49(1 \mathrm{H}, \mathrm{ddd}, J$ 13.9, 9.9, 6.6, C(2') $\left.H_{\mathrm{A}}\right), 2.59-2.72\left(2 \mathrm{H}, \mathrm{m}, \mathrm{C}(4) H_{\mathrm{A}}, \mathrm{C}\left(2^{\prime}\right) H_{\mathrm{B}}\right), 2.84$ (1H, ddd, $J$ 17.6, 11.7, 5.2, C(4)HB $), 2.90$ (3H, s, NMe), 3.24-3.30 $(1 \mathrm{H}, \mathrm{m}, \mathrm{C}(2) \mathrm{H}), 3.87$ (3H, s, OMe), $5.56(1 \mathrm{H}, \mathrm{s}, \mathrm{OH}), 6.52(1 \mathrm{H}$, app d, $J$ 8.1, C(8)H), $6.59(1 \mathrm{H}, \mathrm{td}, J 7.3,1.0$, C(6)H), $6.66(1 \mathrm{H}, \mathrm{dd}, J 8.2$, 2.1, C(6")H), 6.75-6.79 (2H, m, C(2")H, C(5")H), 6.98 (1H, app d, $J$ 7.3, C(5)H), $7.08\left(1 \mathrm{H}, \mathrm{td}, J\right.$ 7.3, 1.2, C(7)H); $\delta_{\mathrm{C}}\left(100 \mathrm{MHz}, \mathrm{CDCl}_{3}\right)$ $23.5(C(4)), 24.3(C(3)), 31.6\left(C\left(2^{\prime}\right)\right), 32.9\left(C\left(1^{\prime}\right)\right), 38.0(\mathrm{NMe}), 56.0$ (OMe), $58.2(C(2)), 110.5(C(8)), 110.6(C(5 ")), 114.5(C(2 ")), 115.3$ (C(6)), 119.5 (C(6")), 121.7 (C(4a)), $127.1(C(7)), 128.6(C(5)), 135.3$ (C(1")), 144.7 (C(4")), $145.3(C(8 \mathrm{a})), 145.5(C(3 ")) ; \mathrm{m} / \mathrm{z}\left(\mathrm{ESI}^{+}\right) 298$ $\left([\mathrm{M}+\mathrm{H}]^{+}, 100 \%\right)$; HRMS $\left(\mathrm{ESI}^{+}\right) \quad \mathrm{C}_{19} \mathrm{H}_{24} \mathrm{NO}_{2}{ }^{+}\left([\mathrm{M}+\mathrm{H}]^{+}\right)$requires 298.1802; found 298.1800.

(R)-N(1)-Benzyl-2-[2'-oxo-2'-(3' '-methoxy-4' '-tertbutyldimethylsilyloxyphenyl)ethyl]-1,2,3,4-tetrahydroquinoline 16. Step 1. Preparation of O-tert-butyldimethylsilyl-4-bromoguaiacol 14. tert-Butyldimethylsilyl chloride $(1.50 \mathrm{~g}, 10.0 \mathrm{mmol})$ was added to a stirred solution of 4-bromoguaiacol $(2.00 \mathrm{~g}, 9.85 \mathrm{mmol})$ and imidazole (1.36 g, $20.0 \mathrm{mmol})$ in DMF (5 mL) at rt and the resultant solution was stirred at rt for $16 \mathrm{~h} . \mathrm{H}_{2} \mathrm{O}(15 \mathrm{~mL})$ was then added and the resultant mixture was extracted with hexane $(5 \times 15 \mathrm{~mL})$. The combined organics were dried and concentrated in vacuo to give $\mathbf{1 4}$ as a colourless oil (2.98 g, 95\%); ${ }^{28} \delta_{\mathrm{H}}\left(400 \mathrm{MHz}, \mathrm{CDCl}_{3}\right) 0.14(6 \mathrm{H}, \mathrm{s}$, $\mathrm{SiMe}$ ), 0.98 (9H, s, SiCMe 3 ), 3.79 (3H, s, OMe), 6.71 (1H, d, $J$ 8.3, C(6)H), 6.93 (1H, dd, $J$ 8.3, 2.4, C(5)H), 6.95 (1H, d, J 2.4, C(3)H).

Step 2. $n$-BuLi (2.3 M in hexanes, $2.4 \mathrm{~mL}, 5.4 \mathrm{mmol}$ ) was added dropwise to a stirred solution of $\mathbf{1 4}(1.71 \mathrm{~g}, 5.39 \mathrm{mmol})$ in THF (19 $\mathrm{mL}$ ) at $-78{ }^{\circ} \mathrm{C}$ and the resultant solution was stirred at $-78{ }^{\circ} \mathrm{C}$ for 30 min. A solution of $8(250 \mathrm{mg}, 0.771 \mathrm{mmol})$ in THF $(2 \mathrm{~mL})$ at $-78{ }^{\circ} \mathrm{C}$ was then added and the resultant solution was stirred at $-78^{\circ} \mathrm{C}$ for 90 min. Satd aq $\mathrm{NH}_{4} \mathrm{Cl}(4 \mathrm{~mL})$ was then added and the resultant mixture was allowed to warm to rt and concentrated in vacuo. The residue was partitioned between $\mathrm{CH}_{2} \mathrm{Cl}_{2}(20 \mathrm{~mL})$ and $\mathrm{H}_{2} \mathrm{O}(20 \mathrm{~mL})$, and the organic layer was dried and concentrated in vacuo. Purification via flash column chromatography (eluent $30-40{ }^{\circ} \mathrm{C}$ petrol/ $\mathrm{Et}_{2} \mathrm{O} / \mathrm{NH}_{4} \mathrm{OH}$, 90:9:1) gave 16 as a yellow oil (239 mg, 62\%); $[\alpha]_{\mathrm{D}}^{25}-11.6$ (c 1.0 in $\left.\mathrm{CHCl}_{3}\right) ; v_{\max } 2953,2930,2857,1672 ; \delta_{\mathrm{H}}\left(400 \mathrm{MHz}, \mathrm{CDCl}_{3}\right) 0.17$ (6H, s, SiMe $)$ ) 0.99 (9H, s, SiCMe 3 ), 1.98 (1H, ddt, $J$ 13.3, 5.7, 2.8, $\left.\mathrm{C}(3) H_{\mathrm{A}}\right), 2.10\left(1 \mathrm{H}, \mathrm{tt}, J 13.3,4.9, \mathrm{C}(3) H_{\mathrm{B}}\right), 2.74-2.82(1 \mathrm{H}, \mathrm{m}$, C(4) $\left.H_{\mathrm{A}}\right), 2.97$ (1H, ddd, $J$ 17.1, 13.3, 5.7, C(4) $\left.H_{\mathrm{B}}\right), 3.13(1 \mathrm{H}, \mathrm{dd}, J$ 16.1, 8.3, C(1') $\left.H_{\mathrm{A}}\right), 3.21\left(1 \mathrm{H}, \mathrm{dd}, J 16.1,5.1, \mathrm{C}\left(1^{\prime}\right) H_{\mathrm{B}}\right), 3.81(3 \mathrm{H}, \mathrm{s}$, OMe), 4.16-4.22 (1H, m, C(2)H), $4.48\left(1 \mathrm{H}, \mathrm{d}, J 17.1, \mathrm{NCH}_{\mathrm{A}} \mathrm{H}_{\mathrm{B}} \mathrm{Ph}\right)$, $4.55\left(1 \mathrm{H}, \mathrm{d}, J\right.$ 17.1, $\left.\mathrm{NCH}_{\mathrm{A}} \mathrm{H}_{\mathrm{B}} \mathrm{Ph}\right), 6.45(1 \mathrm{H}, \mathrm{d}, J$ 8.3, C(8)H), 6.62 $(1 \mathrm{H}, \mathrm{td}, J$ 7.3, 0.9, C(6)H), 6.83 (1H, d, $J$ 8.3, C(5")H), 6.95-7.00 $(1 \mathrm{H}, \mathrm{m}, \mathrm{C}(7) H), 7.04(1 \mathrm{H}, \mathrm{d}, J$ 7.3, C(5)H), 7.18-7.31 (5H, m, $P h)$, 7.38 (1H, dd, $J$ 8.3, 2.0, C(6")H), 7.44 (1H, d, J 2.0, C(2")H); $\delta_{\mathrm{C}}(100$ $\left.\mathrm{MHz}, \mathrm{CDCl}_{3}\right)$-4.6 (SiMe 2$), 18.5$ ( $\left.\mathrm{SiCMe}_{3}\right), 23.5(C(4))$, 25.5 (C(3)), 25.6 ( $\left.\mathrm{SiCMe}_{3}\right), 40.8\left(C\left(1^{\prime}\right)\right), 54.1\left(\mathrm{NCH}_{2} \mathrm{Ph}\right), 54.9(C(2))$, $55.4(\mathrm{OMe})$, 111.0 (C(2")), 111.9 (C(8)), $116.0(C(6)), 120.3\left(C\left(5^{\prime \prime}\right)\right), 121.1$ $(C(4 \mathrm{a})), 122.6\left(C\left(6^{\prime \prime}\right)\right), 126.4(o, m-P h), 126.8(p-P h), 127.2(C(7))$, $128.6(o, m-P h), 129.2$ (C(5)), $131.3\left(C\left(1^{\prime \prime}\right)\right), 138.9(i-P h), 144.1$ (C(8a)), 150.2, $151.1\left(C\left(3^{\prime \prime}\right), C\left(4^{\prime \prime}\right)\right), 197.9\left(C\left(2^{\prime}\right)\right) ; \mathrm{m} / \mathrm{z}\left(\mathrm{ESI}^{+}\right) 502$ $\left([\mathrm{M}+\mathrm{H}]^{+}, 100 \%\right)$; HRMS $\left(\mathrm{ESI}^{+}\right) \mathrm{C}_{31} \mathrm{H}_{40} \mathrm{NO}_{3} \mathrm{Si}^{+}\left([\mathrm{M}+\mathrm{H}]^{+}\right)$requires 502.2772; found 502.2768.

(S)-N(1)-Benzyl-2-[2'-(3"'-methoxy-4"'-hydroxyphenyl)ethyl]1,2,3,4-tetrahydroquinoline 17. Step $1 . \mathrm{LiAlH}_{4}(2.4 \mathrm{M}$ in THF, 0.47
$\mathrm{mL}, 0.95 \mathrm{mmol}$ ) was added dropwise to a stirred solution of $\mathbf{1 6}$ (238 $\mathrm{mg}, 0.474 \mathrm{mmol})$ in THF $(3.3 \mathrm{~mL})$ at $0{ }^{\circ} \mathrm{C}$. The resultant solution was heated at reflux for $16 \mathrm{~h}$ and then allowed to cool to rt. $2 \mathrm{M}$ aq $\mathrm{NaOH}$ $(0.5 \mathrm{~mL})$ was then added and the resultant mixture was heated at reflux for $3 \mathrm{~h}$. The resultant mixture was allowed to cool to rt, filtered through Celite ${ }^{\circledR}$ (eluent EtOAc), and the filtrate was concentrated in vacuo.

Step 2. Et 3 SiH $(0.76 \mathrm{~mL}, 4.7 \mathrm{mmol})$ was added to a stirred solution of the residue from the previous step in TFA $(2.3 \mathrm{~mL})$ at $\mathrm{rt}$ and the resultant solution was stirred at $\mathrm{rt}$ for $16 \mathrm{~h}$, then concentrated in vacuo. The residue was partitioned between $\mathrm{CH}_{2} \mathrm{Cl}_{2}(10 \mathrm{~mL})$ and satd aq $\mathrm{NaHCO}_{3}(10 \mathrm{~mL})$, and the organic extract was dried and concentrated in vacuo. Purification via flash column chromatography (eluent 30-40 ${ }^{\circ} \mathrm{C}$ petrol/ $\left./ \mathrm{Et}_{2} \mathrm{O} / \mathrm{NH}_{4} \mathrm{OH}, 50: 50: 1\right)$ gave 17 as a pale yellow oil (109 mg, 62\%); $[\alpha]_{\mathrm{D}}{ }^{25}+0.4$ (c 1.0 in $\left.\mathrm{CHCl}_{3}\right) ; v_{\max } 3502$, 3026, 2934, 1605, $1530 ; \delta_{\mathrm{H}}\left(400 \mathrm{MHz}, \mathrm{CDCl}_{3}\right) 1.78-2.08\left(4 \mathrm{H}, \mathrm{m}, \mathrm{C}(3) \mathrm{H}_{2}, \mathrm{C}\left(1^{\prime}\right) \mathrm{H}_{2}\right), 2.47$ (1H, ddd, $J$ 13.9, 9.9, 6.6, C(2') $\left.H_{\mathrm{A}}\right), 2.64$ (1H, ddd, $J$ 13.9, 10.6, 5.1, $\left.\mathrm{C}\left(2^{\prime}\right) H_{\mathrm{B}}\right), 2.76\left(1 \mathrm{H}, \mathrm{dt}, J 16.5,3.9, \mathrm{C}(4) H_{\mathrm{A}}\right), 2.94(1 \mathrm{H}, \mathrm{ddd}, J 16.5$, 11.9, 6.2, C(4) $\left.H_{\mathrm{B}}\right), 3.38-3.44(1 \mathrm{H}, \mathrm{m}, \mathrm{C}(2) H), 3.84$ (3H, s, OMe), $4.42\left(1 \mathrm{H}, \mathrm{d}, J 17.1, \mathrm{NCH}_{\mathrm{A}} \mathrm{H}_{\mathrm{B}} \mathrm{Ph}\right), 4.57\left(1 \mathrm{H}, \mathrm{d}, J 17.1, \mathrm{NCH}_{\mathrm{A}} H_{\mathrm{B}} \mathrm{Ph}\right)$, $5.45(1 \mathrm{H}, \mathrm{s}, \mathrm{OH}), 6.43(1 \mathrm{H}$, app d, $J$ 8.2, C(8)H), $6.58(1 \mathrm{H}, \mathrm{td}, J$ 7.3, 1.1, C(6)H), $6.61(1 \mathrm{H}, \mathrm{d}, J$ 1.9, C(2")H), $6.63(1 \mathrm{H}, \mathrm{dd}, J$ 8.0, 1.9, C(6")H), $6.81(1 \mathrm{H}, \mathrm{d}, J$ 8.0, C(5")H), 6.93-6.98 (1H, m, C(7)H), 7.02 (1H, d, J 7.3, C(5)H), 7.19-7.32 (5H, m, Ph); $\delta_{\mathrm{C}}\left(100 \mathrm{MHz}, \mathrm{CDCl}_{3}\right)$ $23.6(C(4)), 24.2(C(3)), 31.9\left(C\left(2^{\prime}\right)\right), 33.9\left(C\left(1^{\prime}\right)\right), 54.1\left(\mathrm{NCH}_{2} \mathrm{Ph}\right)$, 55.9 (OMe), $57.3(C(2)), 110.7\left(C\left(2^{\prime \prime}\right)\right), 111.7(C(8)), 114.2\left(C\left(5^{\prime \prime}\right)\right)$, $115.6(C(6)), 120.7\left(C\left(6^{\prime \prime}\right)\right), 121.6(C(4 a)), 126.4(o, m-P h), 126.7(p-$ $P h), 127.1$ (C(7)), 128.5 (o,m-Ph), 128.9 (C(5)), 133.7 (C(1")), 139.2 (i-Ph), $143.6\left(C\left(4^{\prime \prime}\right)\right), 144.5$ (C(8a)), $146.3\left(C\left(3^{\prime \prime}\right)\right) ; \mathrm{m} / \mathrm{z}\left(\mathrm{ESI}^{+}\right) 374$ $\left([\mathrm{M}+\mathrm{H}]^{+}, 100 \%\right)$; HRMS $\left(\mathrm{ESI}^{+}\right) \quad \mathrm{C}_{25} \mathrm{H}_{28} \mathrm{NO}_{2}{ }^{+}\left([\mathrm{M}+\mathrm{H}]^{+}\right)$requires 374.2115; found 374.2118.

(S)-N(1)-Methyl-2-[2'-(3"'-methoxy-4'-hydroxyphenyl)ethyl]1,2,3,4-tetrahydroquinoline [(-)-Galipeine] 18. Pd/C (42 mg, 40\% w/w of substrate 17) was added to a stirred solution of 17 (106 mg, $0.284 \mathrm{mmol}$ ) and formalin (37\% aq HCHO, $0.21 \mathrm{~mL}, 2.84 \mathrm{mmol}$ ) in degassed $\mathrm{MeOH}(5 \mathrm{~mL})$ at $\mathrm{rt}$. The resultant suspension was stirred under $\mathrm{H}_{2}(1 \mathrm{~atm})$ at $\mathrm{rt}$ for $24 \mathrm{~h}$. The resultant suspension was filtered through a short plug of Celite ${ }^{\circledR}$ (eluent $\mathrm{MeOH}$ ) and the filtrate was concentrated in vacuo. Purification via flash column chromatography (eluent $30-40{ }^{\circ} \mathrm{C}$ petrol $/ \mathrm{Et}_{2} \mathrm{O} / \mathrm{NH}_{4} \mathrm{OH}, 66: 33: 1$ ) gave 18 as a colourless oil (59 mg, $70 \%) ;{ }^{1}[\alpha]_{\mathrm{D}}{ }^{25}-22.0\left(c 0.2\right.$ in $\left.\mathrm{CHCl}_{3}\right)$; $[\alpha]_{\mathrm{D}}{ }^{25}-22.3(c$ 1.0 in $\mathrm{CHCl}_{3}$ ); $[\alpha]_{\mathrm{D}}{ }^{25}-14.0$ (c 1.0 in $\left.\mathrm{MeOH}\right) ;[\alpha]_{\mathrm{D}}^{25}-11.9$ (c 1.0 in EtOH); $v_{\max }$ (ATR) 3510, 2935, 2860, 2844, 1602, 1514, 1500; $\delta_{\mathrm{H}}$ $\left(400 \mathrm{MHz}, \mathrm{CDCl}_{3}\right) 1.68-1.78\left(1 \mathrm{H}, \mathrm{m}, \mathrm{C}\left(1^{\prime}\right) \mathrm{H}_{\mathrm{A}}\right), 1.86-2.00(3 \mathrm{H}, \mathrm{m}$, $\left.\mathrm{C}(3) H_{2}, \mathrm{C}\left(1^{\prime}\right) H_{\mathrm{B}}\right), 2.52$ (1H, ddd, $J$ 13.9, 10.2, 6.4, C(2') $\left.H_{\mathrm{A}}\right), 2.62-$ $2.73\left(2 \mathrm{H}, \mathrm{m}, \mathrm{C}(4) H_{\mathrm{A}}, \mathrm{C}\left(2^{\prime}\right) H_{\mathrm{B}}\right), 2.86(1 \mathrm{H}$, ddd, $J$ 17.5, 11.6, 6.1, $\left.\mathrm{C}(4) H_{\mathrm{B}}\right), 2.92$ (3H, s, NMe), 3.26-3.33 (1H, m, C(2)H), 3.88 (3H, s, $\mathrm{OMe}), 5.47(1 \mathrm{H}, \mathrm{s}, \mathrm{OH}), 6.54(1 \mathrm{H}$, app d, $J$ 8.1, C $(8) H), 6.60(1 \mathrm{H}, \mathrm{td}$, $J$ 7.3, 0.9, C(6)H), 6.67-6.71 (2H, m, C(2")H, C(6")H), $6.84(1 \mathrm{H}, \mathrm{d}, J$ 8.5, C(5")H), 6.99 (1H, d, J 7.3, C(5)H), 7.07-7.12 (1H, m, C(7)H); $\delta_{\mathrm{C}}\left(100 \mathrm{MHz}, \mathrm{CDCl}_{3}\right) 23.5(C(4)), 24.3(C(3)), 32.0\left(C\left(2^{\prime}\right)\right), 33.1$ (C(1')), 38.1 (NMe), 55.9 (OMe), 58.3 (C(2)), 110.5 (C(8)), 110.7 $\left(C\left(2^{\prime \prime}\right)\right), 114.2\left(C\left(5^{\prime \prime}\right)\right), 115.3(C(6)), 120.7\left(C\left(6^{\prime \prime}\right)\right), 121.6(C(4 \mathrm{a}))$, $127.1(C(7)), 128.6(C(5)), 133.9\left(C\left(1^{\prime \prime}\right)\right), 143.6(C(4 ")), 145.2$ $(C(8 \mathrm{a})), 146.3\left(C\left(3^{\prime \prime}\right)\right) ; \mathrm{m} / \mathrm{z}\left(\mathrm{ESI}^{+}\right) 298\left([\mathrm{M}+\mathrm{H}]^{+}, 100 \%\right) ; \mathrm{HRMS}$ $\left(\mathrm{ESI}^{+}\right) \mathrm{C}_{19} \mathrm{H}_{24} \mathrm{NO}_{2}{ }^{+}\left([\mathrm{M}+\mathrm{H}]^{+}\right)$requires 298.1802; found 298.1801.

\section{ASSOCIATED CONTENT}

\section{Supporting Information}

The Supporting Information is available free of charge on the ACS Publications website at DOI: Xxxxx.

Copies of ${ }^{1} \mathrm{H}$ and ${ }^{13} \mathrm{C}$ NMR spectra (PDF) $\mathrm{X}$-ray data for structure CCDC 1557564 (CIF)

\section{AUTHOR INFORMATION}

\section{Corresponding Author}

*E-mail: steve.davies@chem.ox.ac.uk. 


\section{Notes}

The authors declare no competing financial interest.

\section{ACKNOWLEDGMENT}

We are indebted to Professor Nicolas Fabre for supplying copies of the NMR spectra of (-)-galipeine.

\section{REFERENCES}

(1) Jacquemond-Collet, I.; Hannedouche, S.; Fabre, N.; Fourasté, I.; Moulis, C. Phytochemistry 1999, 51, 1167.

(2) A subsequent study reported the isolation of galipeine as the racemate; see: Jacquemond-Collet, I.; Benoit-Vical, F.; Mustofa; Valentin, A.; Stanislas, E.; Mallié, M.; Fouraste, I. Planta Med. 2002, 68, 68.

(3) For a review, see: Muñoz, G. D.; Dudley, G. B. Org. Prep. Proc. Int. 2015, 47, 179. For more recent syntheses of these members of this alkaloid family, see Refs 4-10.

(4) Chen, F.; Surkus, A.-E.; He, L.; Pohl, M.-M.; Radnik, J.; Topf, C.; Junge, K.; Beller, M. J. Am. Chem. Soc. 2015, 137, 11718.

(5) Chacko, S.; Ramapanicker, R. J. Heterocyclic Chem. 2015, 52, 1902.

(6) Pappoppula, M.; Cardoso, F. S. P.; Garrett, B. O.; Aponick, A. Angew. Chem. Int. Ed. 2015, 54, 15202.

(7) Saito, K.; Miyashita, H.; Akiyama, T. Chem. Commun. 2015, $51,16648$.

(8) Garg, Y.; Gahalawat, S.; Pandey, S. K. RSC Adv. 2015, 5, 38846.

(9) Wang, Y.; Liu, Y.; Zhang, D.; Wei, H.; Shi, M.; Wang, F. Angew. Chem. Int. Ed. 2016, 55, 3776.

(10) Crisenza, G. E. M.; Dauncey, E. M.; Bower, J. F. Org. Biomol. Chem. 2016, 14, 5820.

(11) Yang, P.-Y.; Zhou, Y.-G. Tetrahedron: Asymmetry 2004, 15,

(12) The temperature at which the specific rotation value was recorded is given as either $15^{\circ} \mathrm{C}$ or $20^{\circ} \mathrm{C}$ within the manuscript.

(13) Taylor, L. L.; Goldberg, F. W.; Hii, K. K. Org. Biomol. Chem. 2012, 10, 4424.

(14) Willand-Charnley, R.; Puffer, B. W.; Dussault, P. H. J. Am. Chem. Soc. 2014, 136, 5821.

(15) Costello, J. F.; Davies, S. G.; Ichihara, O. Tetrahedron: Asymmetry 1994, 5, 1999.

(16) Zhong, H. M.; Cohen, J. H.; Abdel-Magid, A. F.; Kenney, B. D.; Maryanoff, C. A.; Shah, R. D.; Villani, F. J., Jr.; Zhang, F.; Zhang, X. Tetrahedron Lett. 1999, 40, 7721.

(17) Davies, S. G.; Fletcher, A. M.; Lee, J. A.; Roberts, P. M.; Russell, A. J.; Taylor, R. J.; Thomson, A. D.; Thomson, J. E. Org. Lett. 2012, 14, 1672.

(18) Davies, S. G.; Fletcher, A. M.; Roberts, P. M.; Thomson, J. E.; Zimmer, D. Org. Lett. 2017, 19, 1638.

(19) Crystallographic data (excluding structure factors) have been deposited with the Cambridge Crystallographic Data Centre as supplementary publication number CCDC 1557564.

(20) Flack, H. D. Acta Cryst. A 1983, 39, 876.

(21) Flack, H. D.; Bernardinelli, G. J. Appl. Cryst. 2000, 33, 1143.

(22) $\Delta \delta_{\mathrm{X}}=\mid \delta_{\mathrm{X}}$ (data set A) $-\delta_{\mathrm{X}}$ (data set $\left.\mathrm{B}\right) \mid$, where $\mathrm{X}=\mathrm{H}$ or $\mathrm{C}$, for the two relevant data sets $\mathrm{A}$ and $\mathrm{B}$. Herein, data set $\mathrm{A}$ is taken as our values for either $\mathbf{1 3}$ or $\mathbf{1 8}$, as relevant.

(23) The full data comparison is contained within the Supporting Information.

(24) Professor Fabre was "almost sure that the solvent was chloroform".

(25) Pangborn, A. B.; Giardello, M. A.; Grubbs, R. H.; Rosen, R. K.; Timmers, F. J. Organometallics 1996, 15, 1518.

(26) Reich, H. J.; Goldenberg, W. S.; Sanders, A. W.; Jantzi, K. L.; Tzschucke, C. C. J. Am. Chem. Soc. 2003, 125, 3509.

(27) Ramacciotti, A.; Fiaschi, R.; Napolitano, E. Tetrahedron: Asymmetry 1996, 7, 1101.

(28) Ghavimi, B.; Magnus, P. Org. Lett. 2014, 16, 1708. 